\title{
IMPACT DES AMÉNAGEMENTS SUR LA MIGRATION ANADROME C SAUMON ATLANTIQUE (SALMO SALAR L.) SUR LE GAVE DE PAU (FRANCE).
}

\author{
M. CHANSEAU, O. CROZE et M. LARINIER
}

C - CEMAGREF - GHAAPPE, Institut de Mécanique des Fluides, Avenue du Professeur Camille Soula, 31400 Toulouse, France.

\section{RȨ́UMÉ}

Le suivi par radiotélémétrie de la migration anadrome de 114 saumons atlantiques sur le Gave de Pau, réalisé de 1995 à 1997, a permis d'étudier l'impact de 31 des 37 obstacles érigés sur l'axe de migration. Cinq obstacles majeurs ont été identifiés en regard de leur faible perméabilité et/ou des retards qu'ils induisent ainsi que de leur position sur l'axe. La transparence de ces 5 aménagements permettrait à plus de $80 \%$ (contre seulement $13 \%$ dans la situation actuelle) des saumons de parvenir sur de bonnes zones de frayères. L'accumulation des retards au niveau de chaque obstacle peut empêcher une proportion non négligeable des poissons de parvenir à temps sur les meilleures zones de frayères, notamment ceux qui se présentent à l'automne sur le gave, ou ceux qui, ralentis par les obstacles, effectuent un arrêt estival sur la partie aval du cours d'eau. Les seuils de stabilisation en enrochements de faible hauteur $(<1,5 \mathrm{~m})$ ont été perméables à la migration, la plupart des poissons ayant été retardés moins de 24 heures. Les seuils de hauteur $(>2,5 \mathrm{~m})$ supérieure ont eu des impacts variables, imputables en grande partie au type de passe à poissons. Les passes à bassins et les rivières de contournement ont été les dispositifs de franchissement les plus performants. Les passés à ralentisseurs à chevrons épais se sont révélées peu performantes en raison de leur faible débit d'alimentation et de leur grande sensibilité aux variations des niveaux d'eau amont. L'impact des aménagements hydroélectriques sans dérivation dépend de l'emplacement de l'entrée de la passe à poissons et de son débit d'alimentation : des effets minimums sur la migration ont été observés lorsque les ouvrages étaient équipés d'un dispositif mobilisant des débits importants (2-4 $\left.\mathrm{m}^{3} / \mathrm{s}\right)$ et dont l'entrée se situait dans le canal de fuite. L'impact des aménagements hydroélectriques munis d'une dérivation, tous équipés d'un dispositif de franchissement au niveau des barrages de prise d'eau, dépend des débits réservés dans les parties court-circuitées. Ces bras deviennent très attractifs pour les poissons lorsque leur débit est supérieur à $500 \mathrm{l} / \mathrm{s} / \mathrm{m}$ de largeur au niveau de la confluence avec les canaux de fuite. La grande majorité des franchissements d'obstacles $(85,6 \%)$ a eu lieu en pleine journée. Seules les rivières de contournement assurent un passage nocturne important et semblent respecter davantage les rythmes naturels de migration du saumon.

Mots-clés : saumon atlantique, migration anadrome, aménagement hydroélectrique, seuil, passe à poissons, radio-pistage. 


\section{THE IMPACT OF OBSTACLES ON THE PAU RIVER (FRANCE) ON THE UPSTREAII MIGRATION OF RETURNING ADULT ATLANTIC SALMON (SALMO SALAR L.).}

\section{ABSTRACT}

Radio-tracking of 114 returning adult Atlantic salmon on the Pau River during a 3-year study (1995-1997) was used to determine the impact of 31 of the 37 obstacles built on the river. Five obstacles located downstream of spawning areas have a major effeci on upstream migration with respect to the proportion of fish being able to pass over and the migration delays. An improvement in the facilities allowing fish to pass over these obstructions would enable more than $80 \%$ of them (as opposed to $13 \%$ currently) to reach good spawning grounds. Migration delays can prevent a large proportion of the population from reaching the spawning grounds in time, and this is especially true for fish which enter the river later in autumn or fish which stop their migration for a « quiescent period " in the lower part of the river after being delayed by several obstacles. The lowest weirs $(<1.5 \mathrm{~m})$ seem not to delay fish migration with most of the fish being able to pass over within 24 hours. Higher weirs $(>2.5 \mathrm{~m}$ ) have variable effects on migration depending on the type of fishpass. Pool passes and natural bypass channels are the most efficient. Denil fishpass with floor baffles are not very efficient and this is mainly due to limited operational flow and higher sensitivity to upstream flow level variations. Effects of hydroelectric power plants without diversion depend on the location of fish pass entrances and the flow in the fishway : minimum effects on migration have been observed at plants fitted with fish passes with significant flow $\left(2-4 \mathrm{~m}^{3} / \mathrm{s}\right)$ located in the tailrace. The effect of plants with diversion canal, all fitted with fish passes at dam, are related to the restricted flow in the by-passed section of the river. Dam flow discharge with values greater than $500 \mathrm{l} / \mathrm{s} / \mathrm{m}$ of width at the confluence with the tailrace attract fish. Most upstream passing of obstacles $(85.6 \%)$ occurs during the day. Only natural bypass channels enable a significant proportion of fish to pass over the obstacles at night.

Key-words : Atlantic salmon, upstream migration, hydroelectric power plant, weir, fish pass, radio-tracking.

\section{INTRODUCTION}

Il y a moins d'un siècle, les eaux du Gave de Pau étaient encore largement fréquentées par le saumon atlantique (Salmo salar). Les zones de frayères s'étendaient de Bérenx à l'amont de Lourdes, soit sur près de $100 \mathrm{~km}$. Mais le développement industriel et surtout la construction de barrages ont réduit le saumon à l'état de relique. Les populations de saumons se sont effondrées à partir de la construction de barrages, au niveau de la ville d'Orthez, qui n'étaient pas au départ équipés de passes à poissons (MARTY, 1984). Cette régression s'est encore intensifiée au fil des années suite à l'édification de nouveaux obstacles majeurs, comme Baigts, et à la transformation de moulins à grains en microcentrales qui s'est traduite par une augmentation conséquente des débits dérivés. La construction du barrage d'Artix en 1958, non équipé de passe à poissons, condamne définitivement l'accès aux frayères amont et aboutit à la quasi-disparition du saumon sur le Gave de Pau.

Plus récemment, les extractions intensives de matériaux alluvionnaires dans le lit mineur du gave au cours des années 1970-1980 ont entraîné de profonds bouleversements dans la morphologie du cours d'eau, en particulier un enfoncement du lit qui a conduit à la construction de plusieurs seuils de stabilisation constituant autant d'obstacles potentiels à la migration. 
Dans le cadre du plan de restauration des salmonidés grands migrateurs et des obligations réglementaires, l'aménagement de dispositifs de franchissement a été réalisé sur la plupart des obstacles non équipés. Si la réouverture totale du Gave de Pau paraît maintenant réalisée, il convenait cependant de vérifier la franchissabilité des différents ouvages, en particulier de certains dispositifs de conception ancienne ou d'implantation cor iestable dont l'efficacité pouvait être mise en doute.

Une étude de radiotélémétrie a été réalisée sur trois ans, de 1995 à 1997, afin de délerminer l'impact des différents obstacles sur la migration anadrome des saumons sur l'ensemble de l'axe.

\section{LE AVE DE PAU}

Le Gave de Gavarnie, naissant du cirque du même nom et grossi par les Gaves de Cauterets, de Luz et d'Azun devient le Gave de Pau à hauteur d'Argelès. Il s'écoule ainsi sur $175 \mathrm{~km}$, d'abord sud-nord des sources jusqu'à Lourdes, puis s'oriente sud-est/nord-ouest pour venir confluer avec le Gave d'Oloron puis l'Adour avant de rejoindre l'Océan Atlantique au niveau de Bayonne (Figure 1).

Le bassin du Gave de Pau est soumis à la double influence du climat océanique et de la proximité du massif pyrénéen. Le régime du cours d'eau, de type nivo-pluvial, est marqué par des précipitations annuelles importantes et l'apport prononcé de fonte des neiges : une période d'étiage en fin d'été (août et septembre), d'eaux de fonte d'avril à juin et de fortes précipitations pluviales en décembre. Les affluents de coteaux rive gauche et rive droite se caractérisent par un régime de type essentiellement pluvial, avec des crues violentes et des étiages estivaux très sévères.

Il existe deux stations de mesure des débits qui sont situées, l'une dans la partie amont du secteur d'étude au pont de Rieulhès (bassin versant $1120 \mathrm{~km}^{2}$ ), à l'aval immédiat de Lourdes, et l'autre dans la partie aval du secteur d'étude, au pont de Bérenx (bassin versant $1120 \mathrm{~km}^{2}$ ). Les modules interannuels sont respectivement de $43 \mathrm{~m}^{3} / \mathrm{s}$ et $79 \mathrm{~m}^{3} / \mathrm{s}$ pour la période 1973-1997. La Figure 2 présente les débits moyens mensuels au niveau des deux stations.

\section{OBSTACLES À LA MIGRATION}

II existe aujourd'hui trente-huit obstacles sur la partie du gave classée pour le saumon et la truite de mer. Les 31 obstacles situés entre le barrage de Puyoo, premier obstacle important sur l'axe, et l'aménagement du Lac des Gaves, qui constitue pratiquement la limite amont de l'aire historique de répartition du saumon sur le gave, ont fait l'objet d'un suivi dans le cadre de la présente étude (Figure 1).

Sur ce secteur existent une douzaine de seuils de stabilisation en enrochements de hauteur variant de $1 \mathrm{~m}$ environ à plus de $3,50 \mathrm{~m}$ (Figure 3 ) et une vingtaine de seuils ou barrages associés à des prises d'eau pour la production d'électricité dont les hauteurs varient de $1 \mathrm{~m}$ environ à plus de $10 \mathrm{~m}$.

Les aménagements hydroélectriques situés sur la partie aval du secteur d'étude sont pour la plupart caractérisés par des débits d'équipement importants $\left(50 \mathrm{~m}^{3 / \mathrm{s}}\right.$ à $110 \mathrm{~m}^{3} / \mathrm{s}$ ) et des dérivations très courtes sinon absentes (Figure 4). 

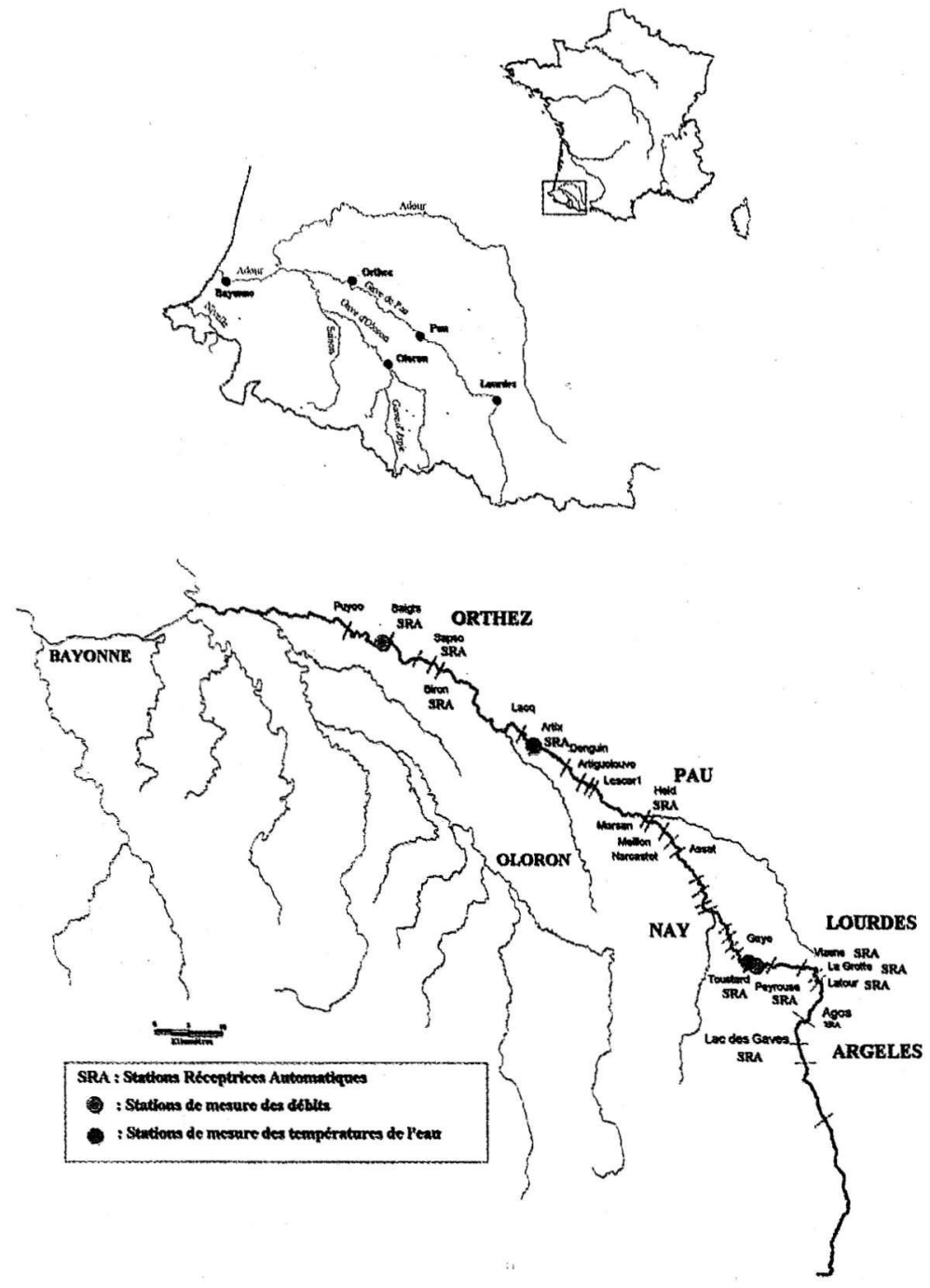

Figure 1

Situation géographique du Gave de Pau et localisation des obstacles.

Figure 1

Geographical situation of Pau river and location of obstacles.

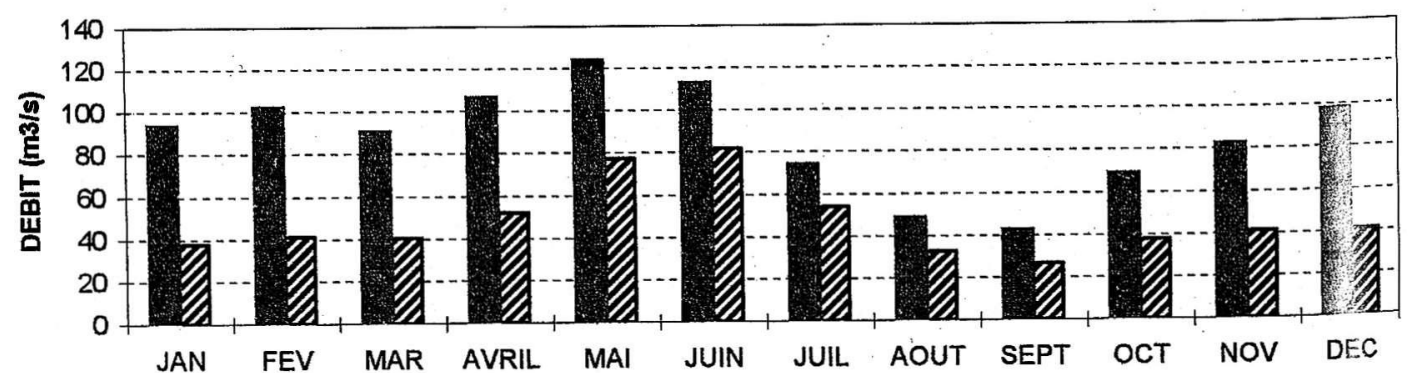

Figure 2

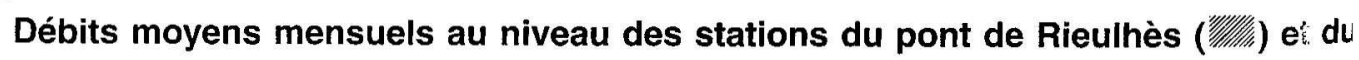
pont de Bérenx (iㅗ).

\section{Figure 2}

Mean monthly flows at Rieulhes bridge station (Y)/n/l) and Berenx brigde staion (피). 

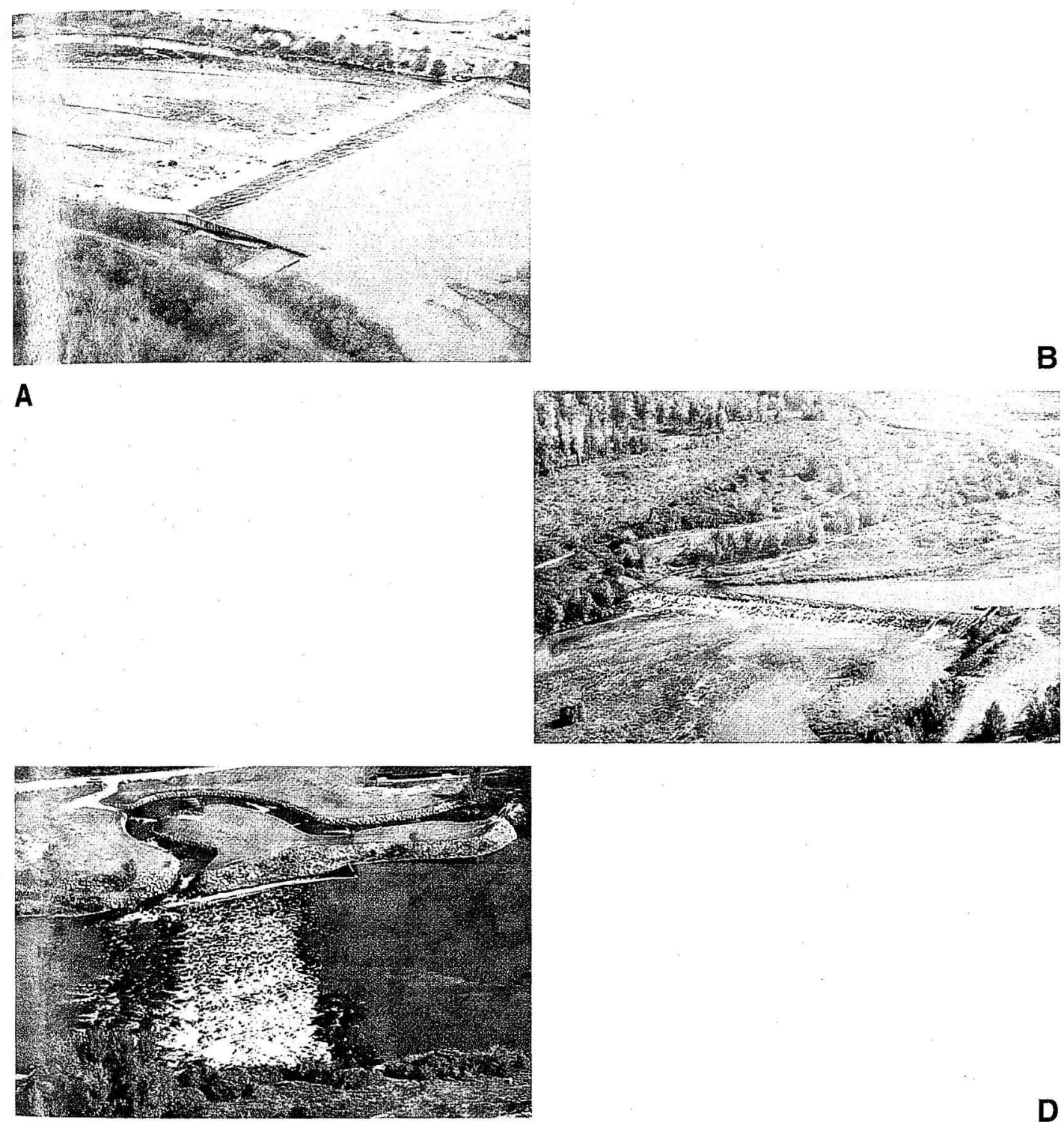

C

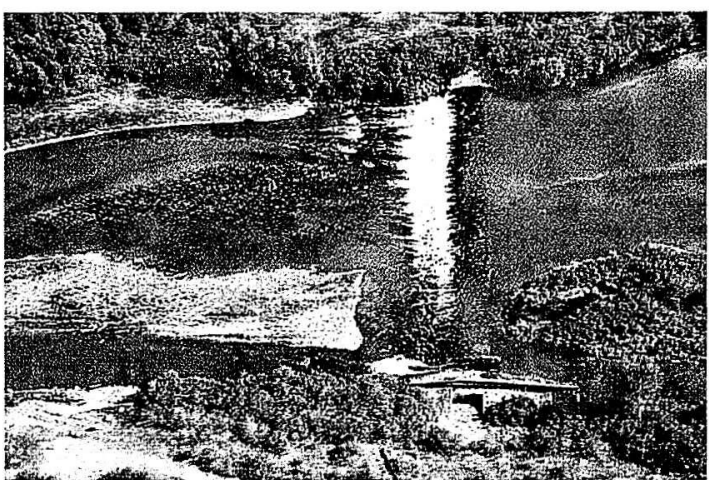

Figure 3

Vue aérienne des seuils en enrochements de Denguin (A), Artiguelouve (B), Meillon (C) et Narcastet (D).

Figure 3

Aerial view of the weirs of Denguin (A), Artiguelouve (B), Meillon (C) and Narcastet (D). 


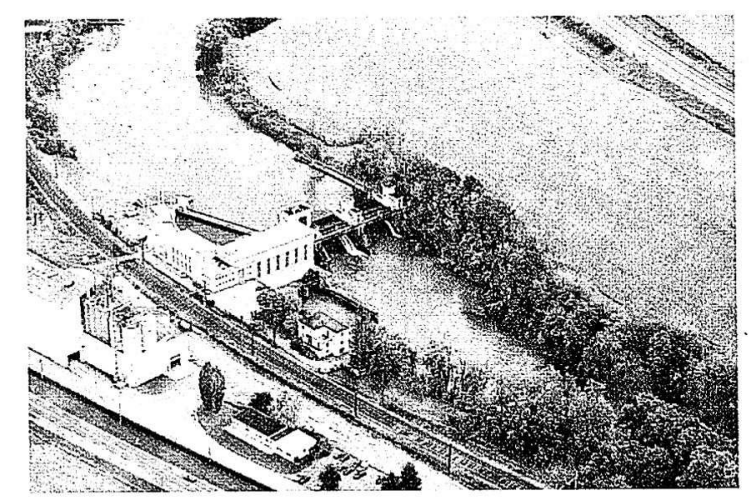

A

C
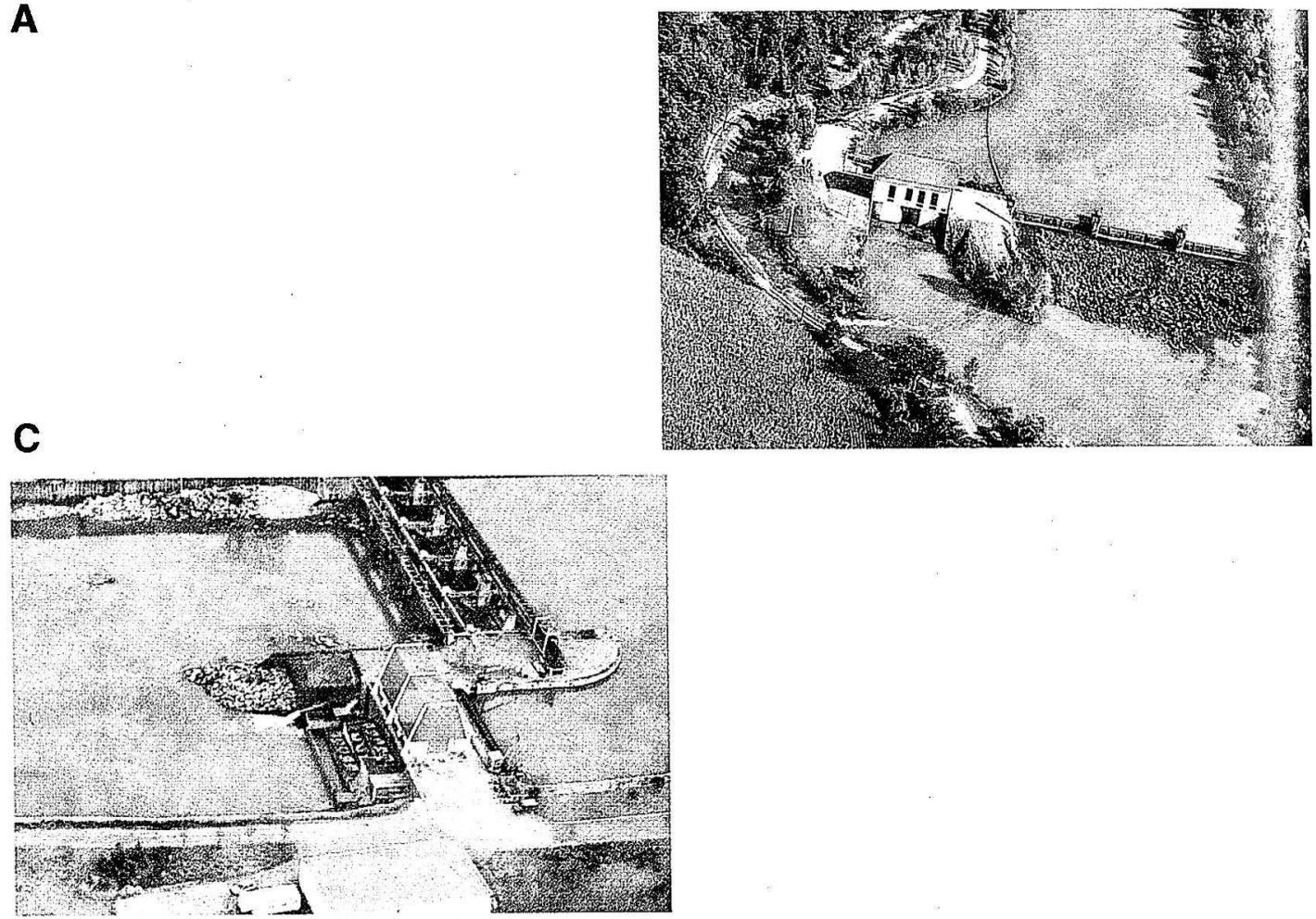

Figure 4

Vue aérienne des aménagements hydroélectriques de Baigts (A), Biron (B) et Artix (C) situés sur la partie aval du Gave de Pau.

Figure 4

Aerial view of Baigts plant (A), Biron plant (B) and Artix plant (C) located in the lower part of the Pau river.

Les ouvrages hydroélectriques situés sur la partie amont du secteur, de hauteul généralement plus faible que les précédents, ont des débits d'équipement inférieurs $\left(12 \mathrm{~m}^{3} / \mathrm{s}\right.$ à $\left.30 \mathrm{~m}^{3} / \mathrm{s}\right)$ mais comportent des dérivations court-circuitant des portions plus ol moins importantes du gave (Figure 5).

En regard de la configuration des aménagements et de leur hauteur, une quinzaine d'ouvrages (dont les ouvrages de Baigts, Sapso, Biron, Artix, Heid, Meillon, Peyrouse, Lac des Gaves) peuvent être considérés, a priori, comme des obstacles totalement et er permanence infranchissables, sinon par le dispositif de franchissement. 
Cinq ouvrages ne sont pas équipés de dispositif de franchissement. II s'agit des se ils en enrochements de Lacq, Lescar 1, Loustau, Gaye et Toustard, tous étant de ha teur modérée.

Les autres obstacles sont équipés d'une ou plusieurs passes à poissons : rivières de ontournement, passes à bassins, passes à ralentisseurs, aménagements rustiques, pr arrages. Certains dispositifs installés sur les seuils de stabilisation (passes à ra tisseurs de fond à chevrons épais) sont destinés à assurer à la fois le franchissement de "obstacle par les poissons et par les canoës-kayaks.
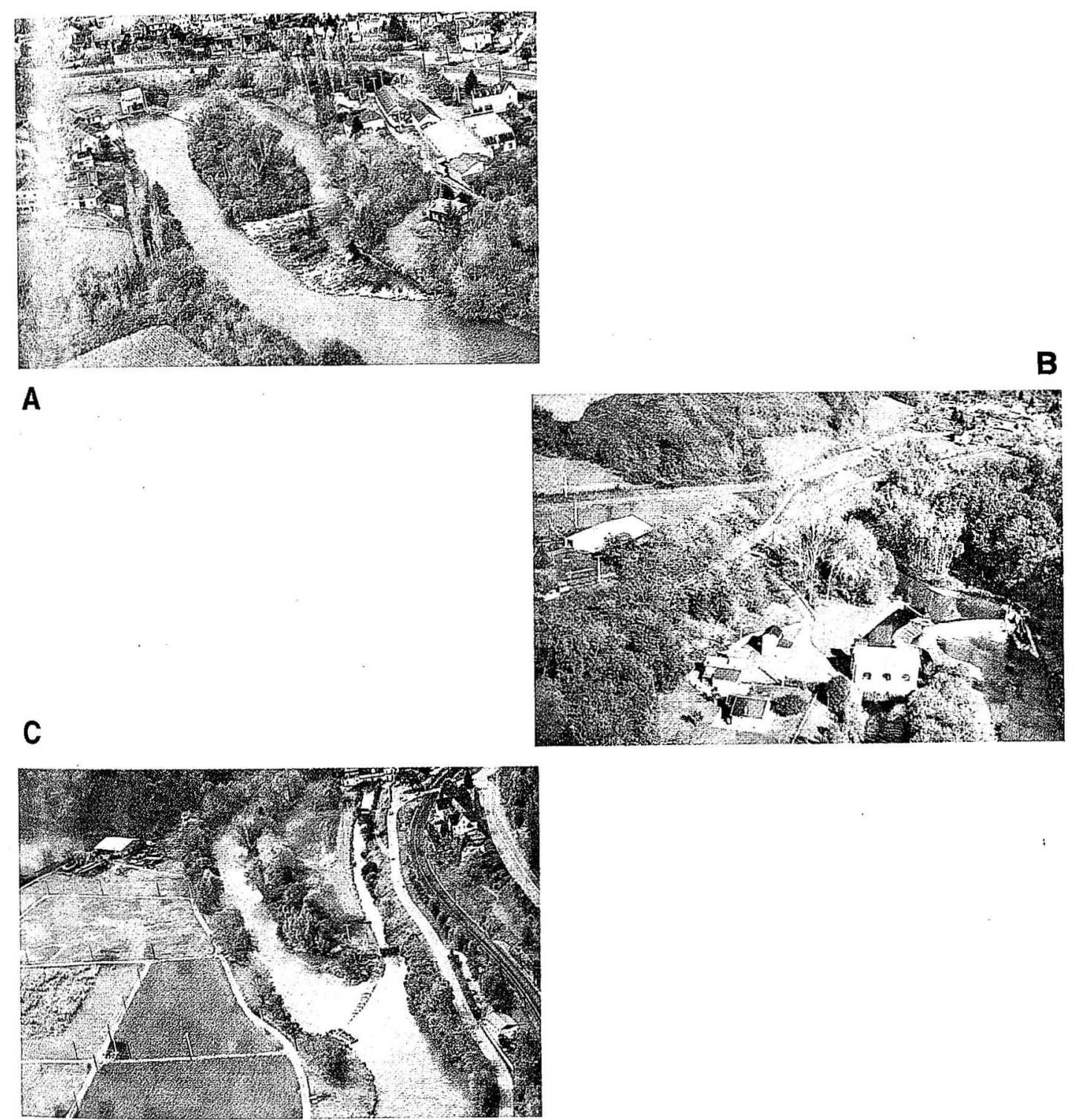

Figure 5

Vue aérienne des aménagements hydroélectriques de Toustard (A), Peyrouse (B) et Vizens (C) situés sur la partie amont du Gave de Pau.

\section{Figure 5}

Aerial view of Toustard plant (A), Peyrouse plant (B) and Vizens plant (C) located in the upper part of the Pau river. 


\section{MATÉRIEL ET MÉTHODES}

\section{Période d'étude}

La migration des saumons sur le Gave de Pau a été étudiée durant 3 anné :s consécutives, de 1995 à 1997. Le piégeage des saumons et le suivi des poisso is radiomarqués ont débuté, selon les années, de la mi-mai à début juillet pour se termirır aux alentours de la mi-décembre.

\section{Débit et température de l'eau}

Les débits ont été relevés aux 2 stations de mesure de Rieulhès et de Bérenx (Figure ).

Des stations d'enregistrement (centrales LOGICAP) des températures de l'eau cont été installées au niveau des aménagements de Toustard et d'Artix (Figure 2).

\section{Capture et marquage des poissons}

Les poissons ont été capturés dans la passe à bassins équipant le barrage de Puyco. Deux fois par jour, le matin et en fin d'après-midi, la passe était fermée afin de contrôler la présence éventuelle de poissons dans le piège. Les saumons piégés étaient anesthésiés à l'aide de phénoxy-2-éthanol dans la proportion de 2,5 $\mathrm{ml}$ pour 10 litres d'eau. Les poissons étaient mesurés (longueur totale), leur sexe déterminé et leur aspect général relevé : couleur de la robe, présence de poux de mer... Les poissons blessés, écaillés ou chétifs étaient écartés.

Lors des 3 années d'étude, 114 saumons ont été marqués en introduisant un émetteur dans leur estomac à l'aide d'un tube pousseur en plastique. Ce marquage stomacal est généralement considéré comme la méthode la mieux adaptée au saumon adulte lors de sa migration en rivière (STASKO et PINCOCK, 1977 ; SOLOMON et STRORETON-WEST, 1983) car elle ne nécessite pas une longue manipulation du poisson et permet une récupération rapide des individus. De plus, la régurgitation est généralement faible (SMITH, CAMPBELL et MacLAINE, 1998).

\section{Transport des saumons}

En raison des difficultés rencontrées par les saumons pour parvenir sur le haut du bassin, et afin d'évaluer l'impact d'un maximum d'obstacles sur la migration anadrome, les poissons marqués ont été relâchés en plusieurs points sur l'axe.

\section{Suivi par radiotélémétrie}

L'ensemble du matériel utilisé est de marque ATS (Advanced Telemetry System). Les émetteurs sont de forme cylindrique $(60 \times 20 \mathrm{~mm} \varnothing)$ et pèsent dans l'air entre 20 el $22,5 \mathrm{~g}$. L'antenne mesure $25 \mathrm{~cm}$ de long. Le poids de l'émetteur représente nettement moins de $1,5 \%$ du poids du poisson. Chaque émetteur a une fréquence propre d'émission comprise entre 48 et $49,9 \mathrm{Mhz}$. Lautonomie minimale de ces émetteurs, garantie par l $\epsilon$ constructeur, est de 220 jours.

Des stations de réception fixes automatiques ont été installées au niveau d'une quinzaine d'obstacles tout au long de l'axe ; elles ont permis d'enregistrer les heures d'arrivée, celles de départ, et par conséquent les durées de présence ainsi que les retards de migration. 
Des contraintes évidentes en ce qui concerne l'alimentation électrique et la prc ection du matériel installé n'ont pas permis d'équiper l'ensemble des aménagements, en articulier les seuils en enrochements. Entre les différents obstacles qui jalonnent le gave et au iveau des obstacles non équipés de postes fixes, le suivi des déplacements des poissons a fó réalisé de façon manuelle (en voiture, à pied et en avion). Dans le souci de recueillir le me imum de données, on s'est efforcé d'obtenir au moins quotidiennement des informations su: la position de chaque poisson radiomarqué. Pour les retards induits par ces arr nagements, seules des fourchettes de valeurs ont pu être estimées. Lorsqu'un poisson a ét ocalisé à l'amont d'un obstacle sans jamais avoir été repéré au pied de l'ouvrage, un retard mo imum a été calculé en tenant compte de sa dernière localisation à l'aval, et de sa première loc. isation à l'amont, ainsi que de la distance séparant ces deux localisations. Dans ce cas, le tard minimum est considéré comme étant inférieur à $24 \mathrm{~h}$. Quand un poisson a été repéré plis ieurs fois au pied d'un obstacle puis à l'amont, le retard minimum correspond au laps de ter: is séparant les premier et dernier repérages.

\section{RE ILTATS}

Une vingtaine d'obstacles sur les 31 étudiés au cours des 3 années ont reçu la visite d'a: moins 10 poissons (jusqu'à 24 saumons), nombre permettant de se faire une idée relativement précise de l'influence de ces ouvrages sur la migration (Tableau I). L'impact géréral des autres aménagements a pu être évalué plus ou moins grossièrement en intégrant la connaissance et l'expertise des sites aux résultats des études télémétriques.

\section{Tableau I}

\section{Caractéristiques des obstacles étudiés.}

\section{Table I}

\section{Characteristics of the studied obstacles.}

\begin{tabular}{|c|c|c|c|c|c|c|c|c|}
\hline OBSTACLES & $\begin{array}{l}\text { INDICE } \\
\text { OMETRIQUE }\end{array}$ & $\begin{array}{c}\text { HAUTEUR } \\
\text { (nuètre) }\end{array}$ & TYPE & USAGE & $\begin{array}{c}\text { DEBIT } \\
\text { TURBINE } \\
\text { MAXIMUM } \\
(\mathrm{m} 3 / \mathrm{s})\end{array}$ & $\begin{array}{c}\text { DISPOSITIF } \\
\text { FRANCHISSEMENT }\end{array}$ & $\begin{array}{l}\text { NOMBRE } \\
\Gamma \text { POISSONS E }\end{array}$ & $\begin{array}{l}F c \\
F \text { FFICACITE }\end{array}$ \\
\hline BAIGTS & 8 & 11 & Burrage & Electricité & 90 & P. à ralentisseurs & 34 & 35 \\
\hline SAPSO & 16.5 & 4.8 & Barrage & Electricité & 48 & P. a ralentisseurs & 19 & 73,7 \\
\hline BIRON & 18,5 & 4 & Barrage & Electricité & 110 & Rivièrce & 20 & 100 \\
\hline LACQ & 35,6 & 1.5 & Seuil & Stabilisation & - & - & 15 & 100 \\
\hline ARTIX & 41 & 6.5 & Barrage & Electricité & 90 & P. à bassins & 16 & 94 \\
\hline DENGUIN & 48.8 & $2.5-3$ & Seuil & Stabilisation & - & P. à ralentisseurs & 15 & 93,3 \\
\hline ARTIGUELOUVE & 54.2 & 2,5 & Sevil & Stabilisation & - & P. a bassins & 14 & 100 \\
\hline LESCAR I & 56,8 & 1.2 & Seuil & Stabilisation & - & - & 14 & 100 \\
\hline MARSAN & 65.4 & 1 & Sevil & Electricié & 4 & - & 13 & 100 \\
\hline HEID & 67.2 & 3 & Seuil & Electricité & 30 & P. à ralentisscurs & 12 & 58,3 \\
\hline MEILLON & 71 & 3.8 & Seuil & Stabilisation & - & Rivietre & 10 & 100 \\
\hline NARCASTET & 71,9 & 2,6 & Scuil & Stabilisation & & P. à ralcntisscurs & 10 & 70 \\
\hline GAYE & 95 & $<1$ & Seuil & Electricité & 3,5 & - & 10 & 100 \\
\hline TOUSTARD & 98,6 & 1 & Seuil & Electricité & 24 & - & 24 & 91.6 \\
\hline PEYROUSE & 102 & 3,5 & Sevil & Electricité & 30 & P. a bassins & 21 & 76,2 \\
\hline VIZENS & 106,9 & 3 & Seuil & Electricité & 30 & P. à bassins & 16 & 62.5 \\
\hline LA GROTTE & 109.1 & 1.5 & Seuil & Electricité & 12 & P. à bassins & 10 & 100 \\
\hline LATOUR & 111,8 & 1 & Scuil & Electricité & 30 & Bassins rustiques & 10 & 80 \\
\hline
\end{tabular}

\section{Rythmes et vitesses de migration}

Soixante-dix valeurs de vitesse de déplacement ont pu être déterminées entre les différents aménagements équipés de stations fixes réceptrices (Figure 6). La majorité des vitesses de déplacement observées sont comprises entre $0,3 \mathrm{~km} / \mathrm{h}$ (7,2 km/jour) et $1,6 \mathrm{~km} / \mathrm{h}(38,4 \mathrm{~km} / \mathrm{jour})$ avec des maxima de $2 \mathrm{~km} / \mathrm{h}$ (48 km/jour). Les vitesses semblent liées à la température de l'eau $(p<0,05)$, la quasi-totalité des déplacements supérieurs à $1 \mathrm{~km} / \mathrm{h}$ se déroulant pour des températures supérieures à $14^{\circ} \mathrm{C}$. 


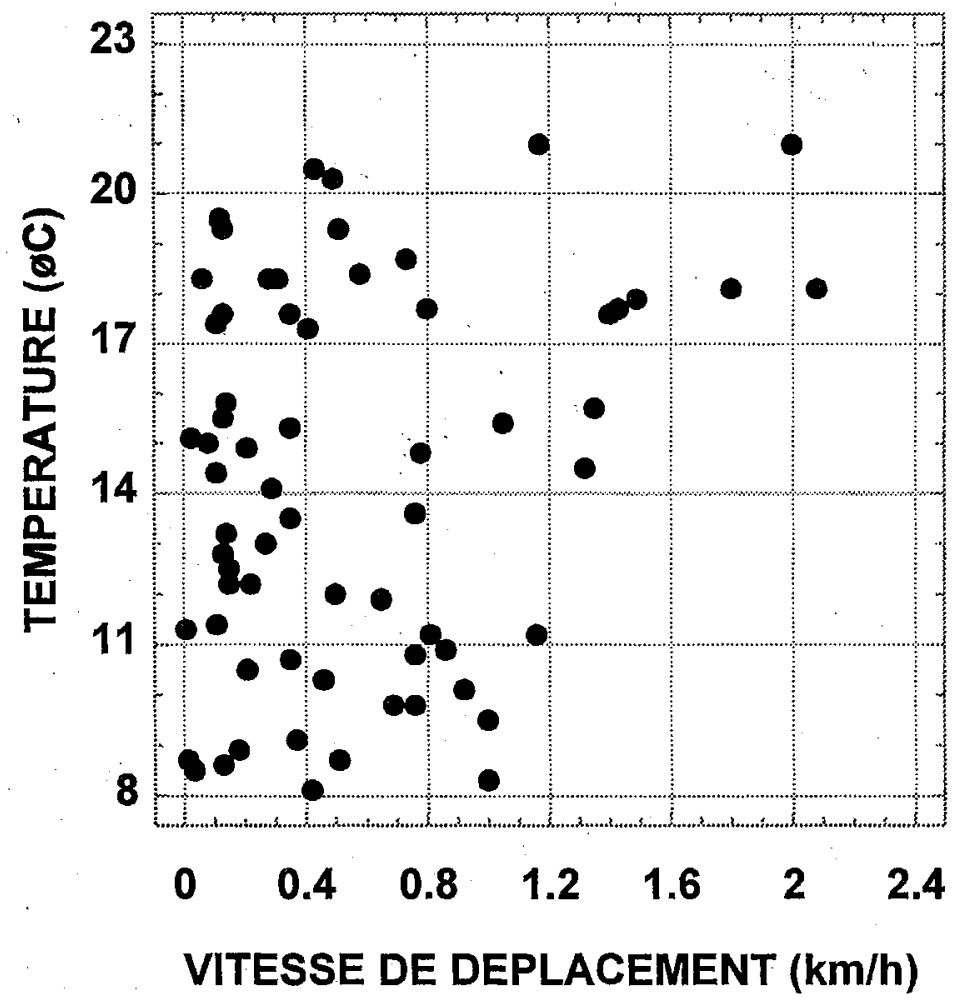

Figure 6

Vitesses de déplacement des poissons entre 2 obstacles équipés de station de réception automatique en fonction des températures de l'eau.

\section{Figure 6}

Swimming speeds of fish between 2 obstacles fitted with automatic listening station according to water temperatures.

Des vitesses de déplacement sur des portions de cours d'eau, comportant plusieurs aménagements, ont été calculées sur trois tronçons distincts.

Le premier tronçon, situé sur le bas du bassin, est compris entre les obstacles de Baigts et de Biron et est long de 10,5 kilomètres. Les 8 poissons qui ont effectué ce parcours ont dû franchir les aménagements de Baigts et de Sapso, deux obstacles majeurs du Gave de Pau. Ce tronçon exerce un impact important sur la migration, les vitesses moyennes de déplacement étant très faibles, généralement inférieures à $0,5 \mathrm{~km} / j o u r$. La majorité des poissons a mis plus de 3 semaines pour parcourir cette distance pour des maximums supérieurs à 2 mois (Figure 7A).

Le second tronçon, d'un linéaire de 48,7 kilomètres, situé immédiatement dans la continuité du premier, couvre la partie moyenne du cours d'eau jusqu'à la ville de Pau qui se situe à l'aval des premières bonnes zones de frayères. II inclut 8 obstacles, pour la plupart des seuils en enrochements, et a été parcouru par 12 saumons. La majorité des poissons a présenté des vitesses moyennes de déplacement comprises entre 2,4 km/jour et $8,4 \mathrm{~km} / \mathrm{jour}$, supérieures à celles du premier tronçon. Trois poissons ont toutefois mis plus de 1 mois, avec un maximum de 100 jours pour un saumon incluant un arrêt estival de près de 70 jours de début août à début octobre (Figure 7B). 

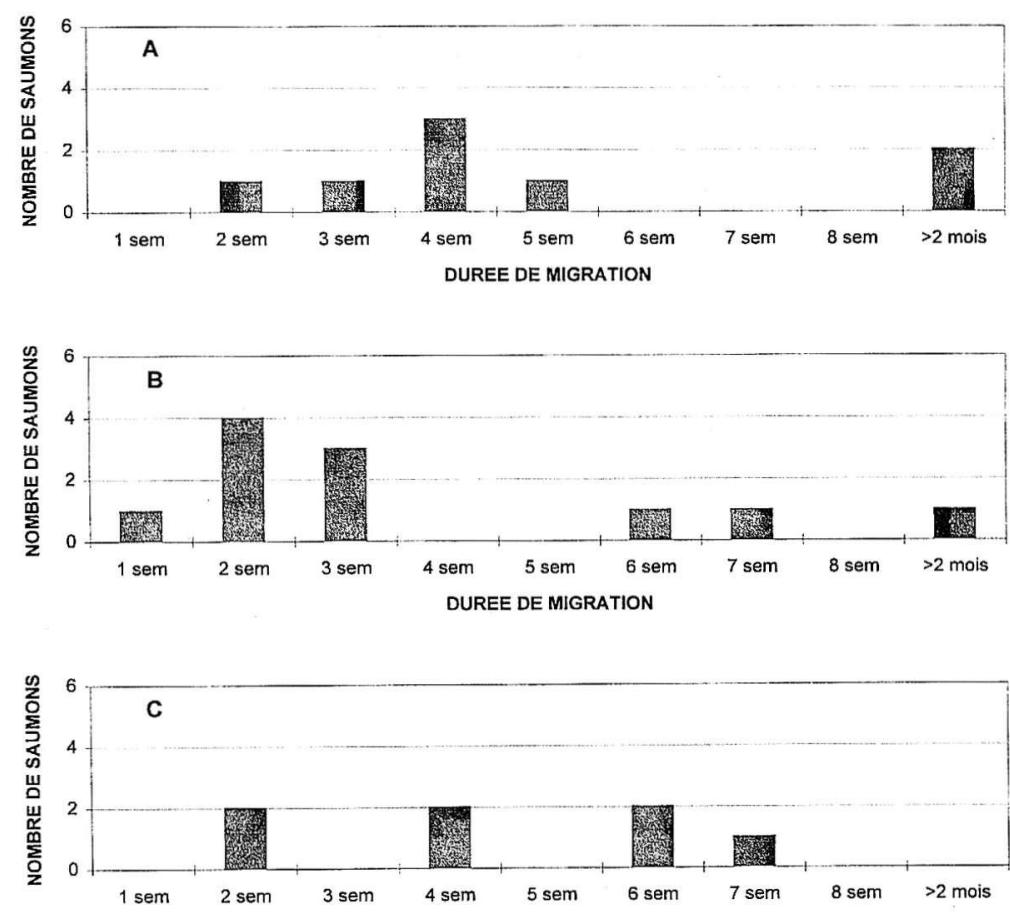

Figure 7A

DUREE DE MIGRATION

Temps mis par les poissons pour parcourir le premier tronçon $(10,5 \mathrm{~km})$ situé entre les aménagements de Baigts et de Biron.

\section{Figure 7B}

Temps mis par les poissons pour parcourir le deuxième tronçon $(48,7 \mathrm{~km})$ situé entre l'aménagement de Biron et la ville de Pau.

\section{Figure 7C}

Temps mis par les poissons pour parcourir le troisième tronçon $(20,3 \mathrm{~km})$ situé entre les aménagements de Toustard et Agos.

\section{Figure 7A}

Progression rates in the first reach $(10.5 \mathrm{~km})$ between Baigts plant and Biron plant.

Figure 7B

Progression rates in the second reach $(48.7 \mathrm{~km})$ between Biron plant and Pau. Figure 7C

Progression rates in the third reach $(20.3 \mathrm{~km})$ between Toustard plant and Agos plant.

Le troisième enfin, situé au niveau des meilleures zones de frayères, sur la partie amont du secteur d'étude, est long de 20,3 kilomètres et inclut 5 aménagements hydroélectriques munis de dérivation, les dispositifs de franchissement étant tous situés au niveau des barrages. Deux groupes de poissons peuvent être individualisés : ceux qui ont mis moins de 27 jours (vitesses comprises entre 0,7 et $1,4 \mathrm{~km} / \mathrm{jour}$ ) pour parcourir le tronçon et ceux qui ont mis plus de 37 jours (vitesses inférieures à $0,5 \mathrm{~km} / \mathrm{jour}$ ) (Figure 7C).

Le suivi des déplacements a montré que la grande majorité des arrêts de migration a été induit par les différents obstacles érigés sur l'axe (Figures 8 et 9 ). Après avoir franchi un ouvrage, les poissons ont la plupart du temps nagé jusqu'à l'aménagement suivant sans marquer de réels arrêts de migration. Dans ces conditions, il était difficile de mettre en évidence un effet quelconque des débits du gave ou de la température de l'eau, seule la présence des obstacles rythmant le déplacement des poissons. 

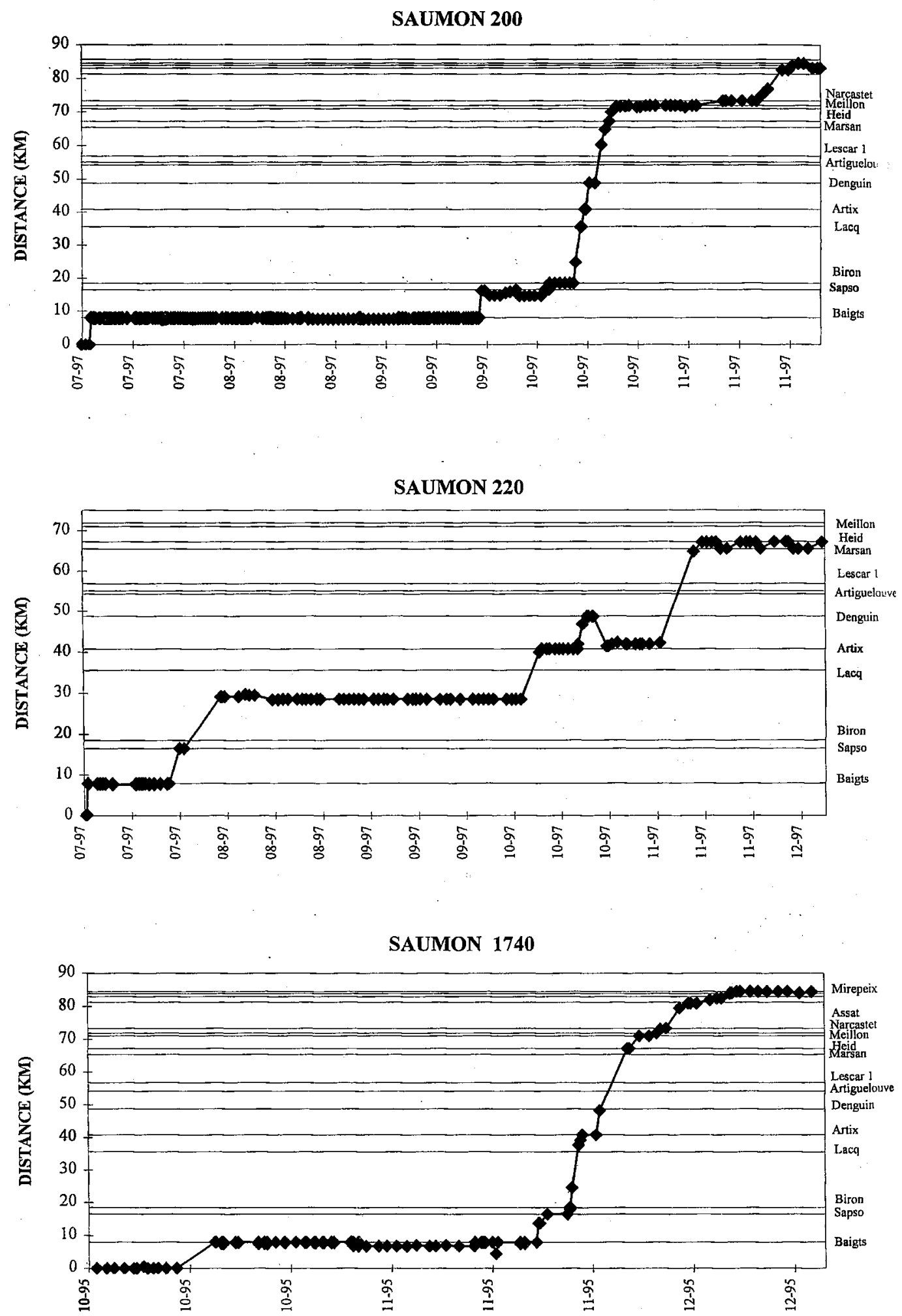

Figure 8

Mouvements des saumons 200, 220 et 1740 sur le Gave de Pau.

Figure 8

The movements of salmon 200,220 and 1740 within the Pau river. 
Buli. Fr. Pêche Piscic. (1999) 353/354 : 211-237 - $223-$
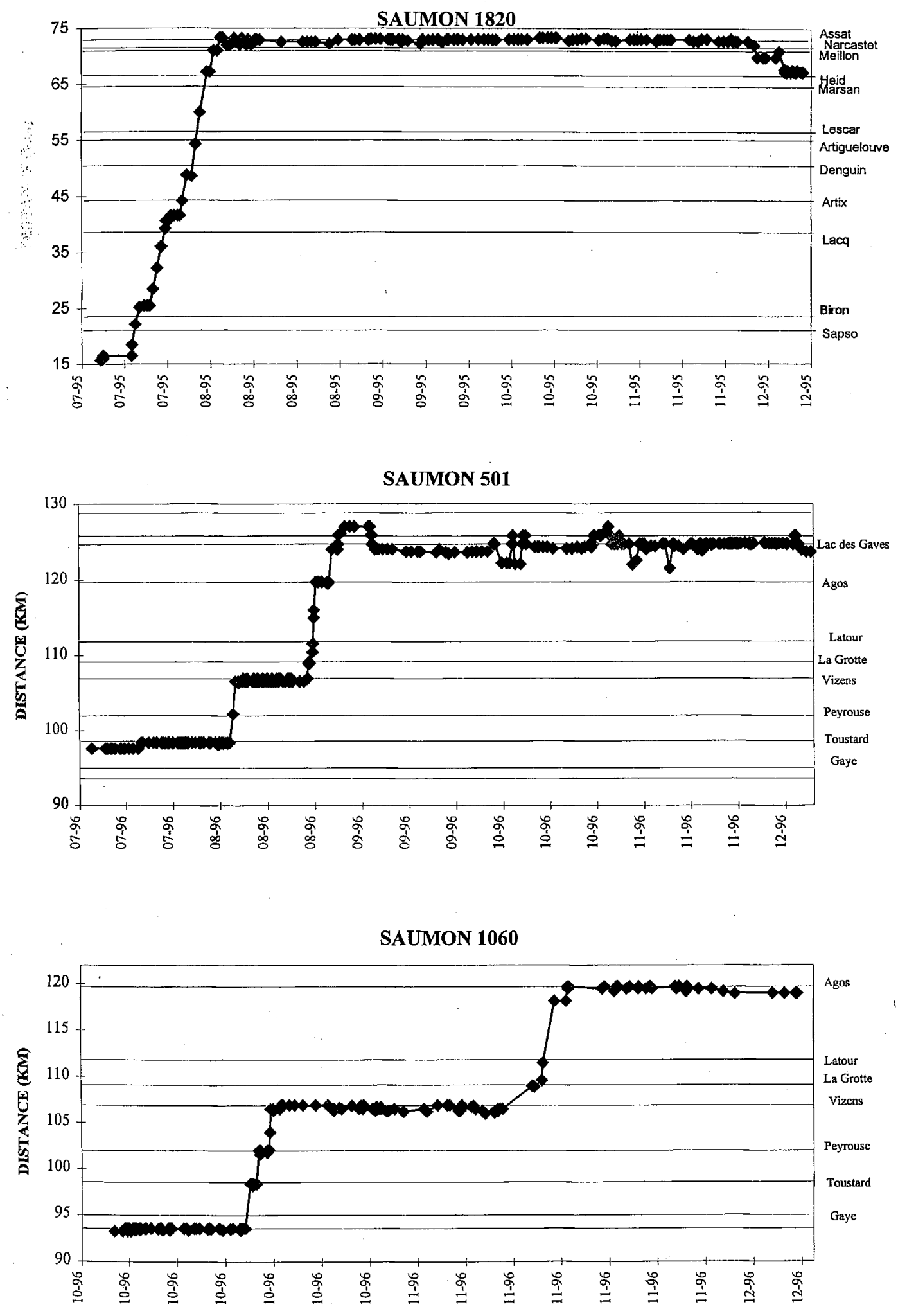

Figure 9

Mouvements des saumons 1820, 501 et 1060 sur le Gave de Pau.

Figure 9

The movements of salmon 1820,501 et 1060 within the Pau river. 
Les déplacements de 6 saumons ont mis en évidence une des composarie: naturelles de la migration des saumons en rivière : les arrêts estivaux. Ces poissons on stoppé leur migration dans un pool ou un profond entre la fin juillet et la mi-acût généralement après une augmentation des températures de l'eau (plus de $2^{\circ} \mathrm{C}$ en $\mathrm{mc} n$ : d'une semaine) associée à une baisse des débits. Ces arrêts ont été compris el ir 40 jours et 75 jours. Les reprises de migration se sont toutes effectuées à l'automne, er rit fin septembre et mi-octobre, après de fortes diminutions des températures (jusqu'à $4^{\circ} \mathrm{C}$ ə moins d'une semaine).

\section{Franchissabilité des obstacles}

La franchissabilité des différents obstacles a été évaluée en termes de retard ¿̀ li migration et de pourcentage de poissons se présentant au pied des ouvrages et parvenar à passer à l'amont. II est intéressant de remarquer que les résultats obtenus lors de 3 années d'étude sont relativement homogènes, et ce malgré des conditions hydrologique différentes.

Les obstacles de faible hauteur, inférieure à $1,5 \mathrm{~m}$, en l'occurrence les seuils $\mathrm{d}$ Lacq, Lescar 1, Marsan et Gaye, non équipés de dispositif de franchissement, laisser passer la totalité des saumons, la grande majorité des poissons franchissant ces obstacle en moins de 24 heures (Figure 10). Les retards induits dans la migration sont faibles, trè peu de saumons ayant d'ailleurs pu être localisés au pied de ces obstacles.

Les seuils en enrochements de hauteur supérieure à $2,5 \mathrm{~m}$, équipés de dispositif d franchissement, s'avèrent plus difficilement franchissables (Figure 11). Deux d'entre eu (Artiguelouve et Meillon) sont totalement perméables et laissent passer tous les poisson en moins d'une semaine, la majorité en moins de 3 jours. Les deux autres (Denguin $\epsilon$ Narcastet) sont plus sélectifs, respectivement $70 \%$ et $90 \%$ des poissons passant l'amont. Près de $30 \%$ des saumons ont été bloqués plus d'une semaine avec des retard dans la migration pouvant dépasser 20 jours.

Les aménagements hydroélectriques situés sur la partie aval du secteur d'étude dépourvus de dérivations importantes, présentent des perméabilités variables Deux d'entre eux (Biron et Artix) laissent passer la quasi-totalité des poissons. Ce aménagements ne retardent pas (Biron) ou peu (Artix) la migration des saumons, le 2 autres ouvrages s'avérant par contre très sélectifs (Baigts, Sapso), entraînant de retards conséquents pouvant dépasser 2 mois (Figure 12).

Les ouvrages hydroélectriques situés sur la partie amont du secteur, caractérisé par des débits d'équipement beaucoup plus faibles (12 à $30 \mathrm{~m}^{3} / \mathrm{s}$ ) et des secteur court-circuités plus importants, présentent eux aussi des perméabilités variable Deux d'entre eux permettent le franchissement de la plupart des individus (Toustare La Grotte), les trois autres aménagements (Peyrouse, Vizens et Latour) se révélar nettement plus sélectifs. Trois de ces ouvrages (Toustard, La Grotte et Latour), ont laiss passer la grande majorité des poissons dans les 3 jours. Les deux autres aménagement (Peyrouse, Vizens), infranchissables sinon par la passe à poissons en regard de let hauteur (supérieure à $3 \mathrm{~m}$ ) et de leur topographie, ont provoqué des retards plu importants, près de la moitié des poissons étant bloquée plus de 3 jours (Figure 13).

\section{Performance des dispositifs de franchissements}

Certains obstacles, comparables tant par leur nature (seuil de stabilisatior aménagement hydroélectrique), leur configuration et l'emplacement des passes poissons, ont présenté des franchissabilités variables. II était tentant de comparer le performances des différents dispositifs de franchissement. 

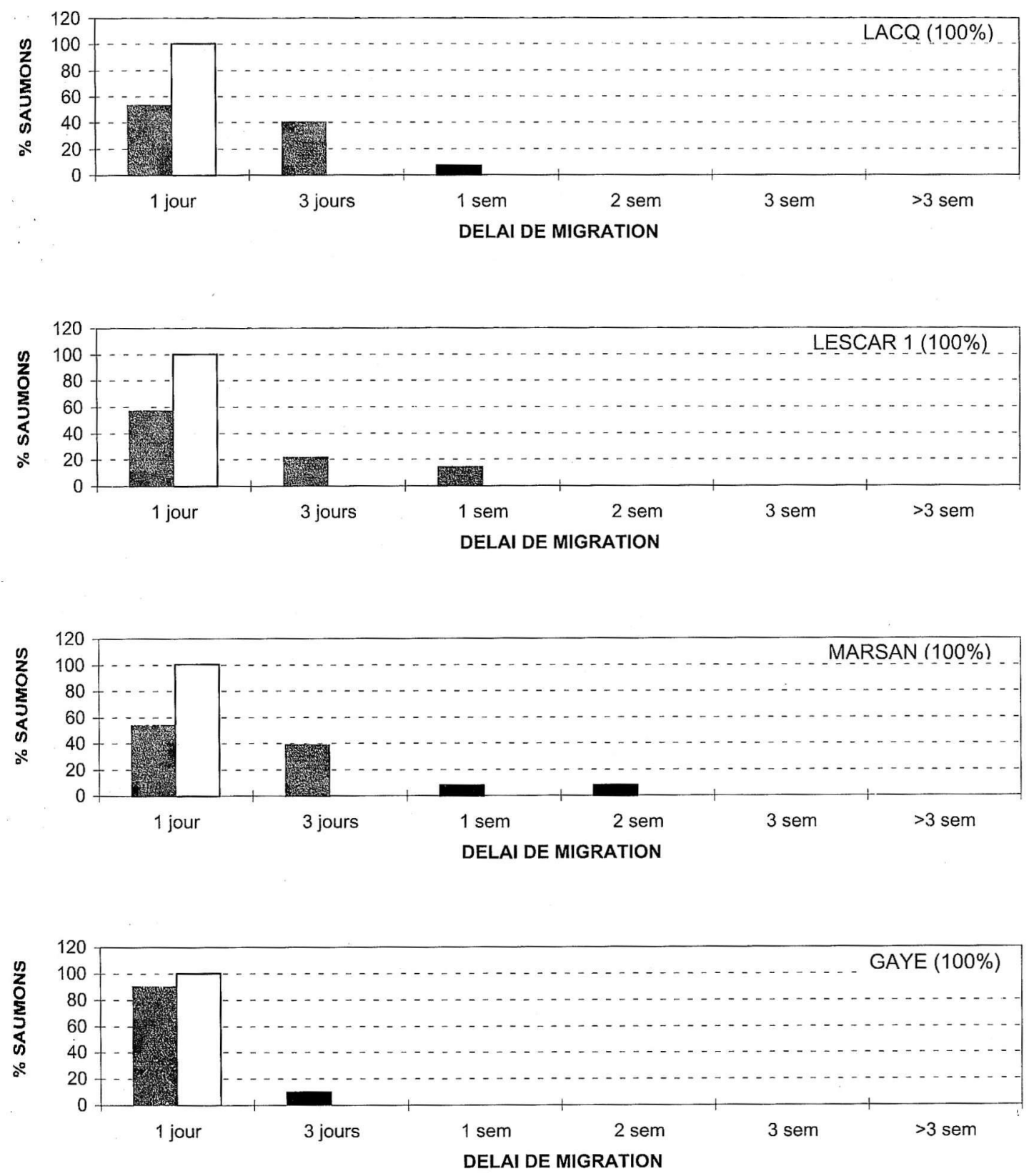

\section{Figure 10}

Délais de migration maximum (1) et minimum ( enrochements de hauteur inférieure à $1,5 \mathrm{~m}$.

\section{Figure 10}

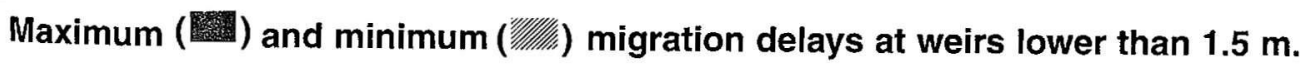



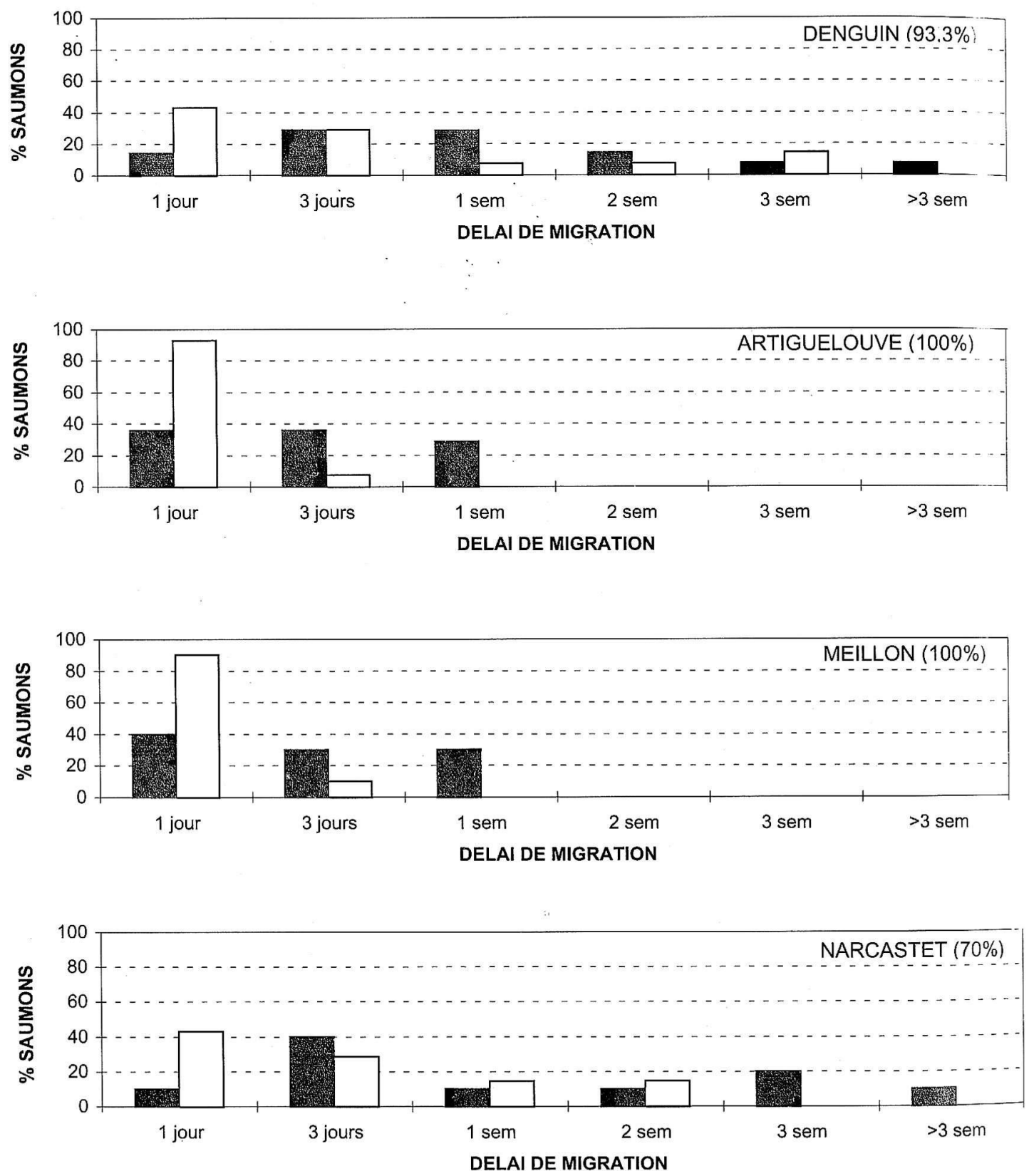

Figure 11

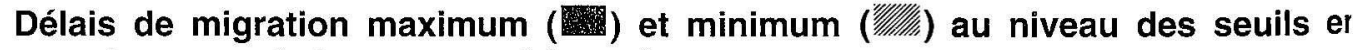
enrochements de hauteur supérieure à $2,5 \mathrm{~m}$.

\section{Figure 11}

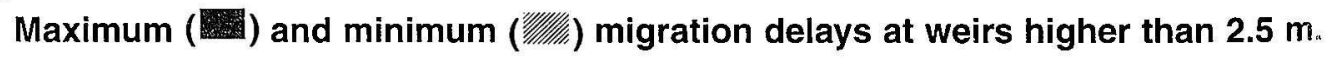



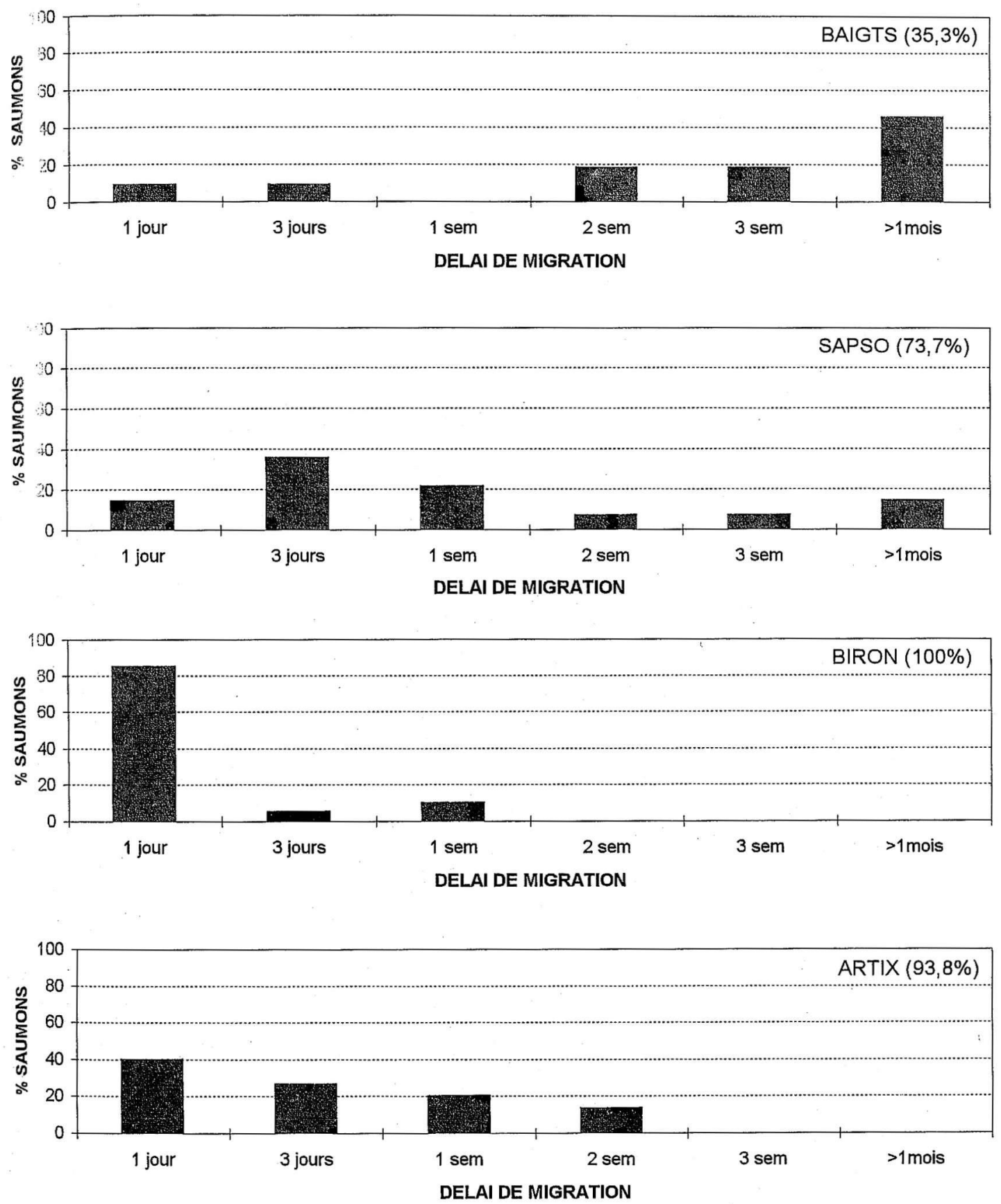

\section{Figure 12}

Délais de migration au niveau des aménagements hydroélectriques situés sur la partie aval du Gave de Pau.

\section{Figure 12}

Migration delays at hydroelectric plants located in the lower part of the river. 

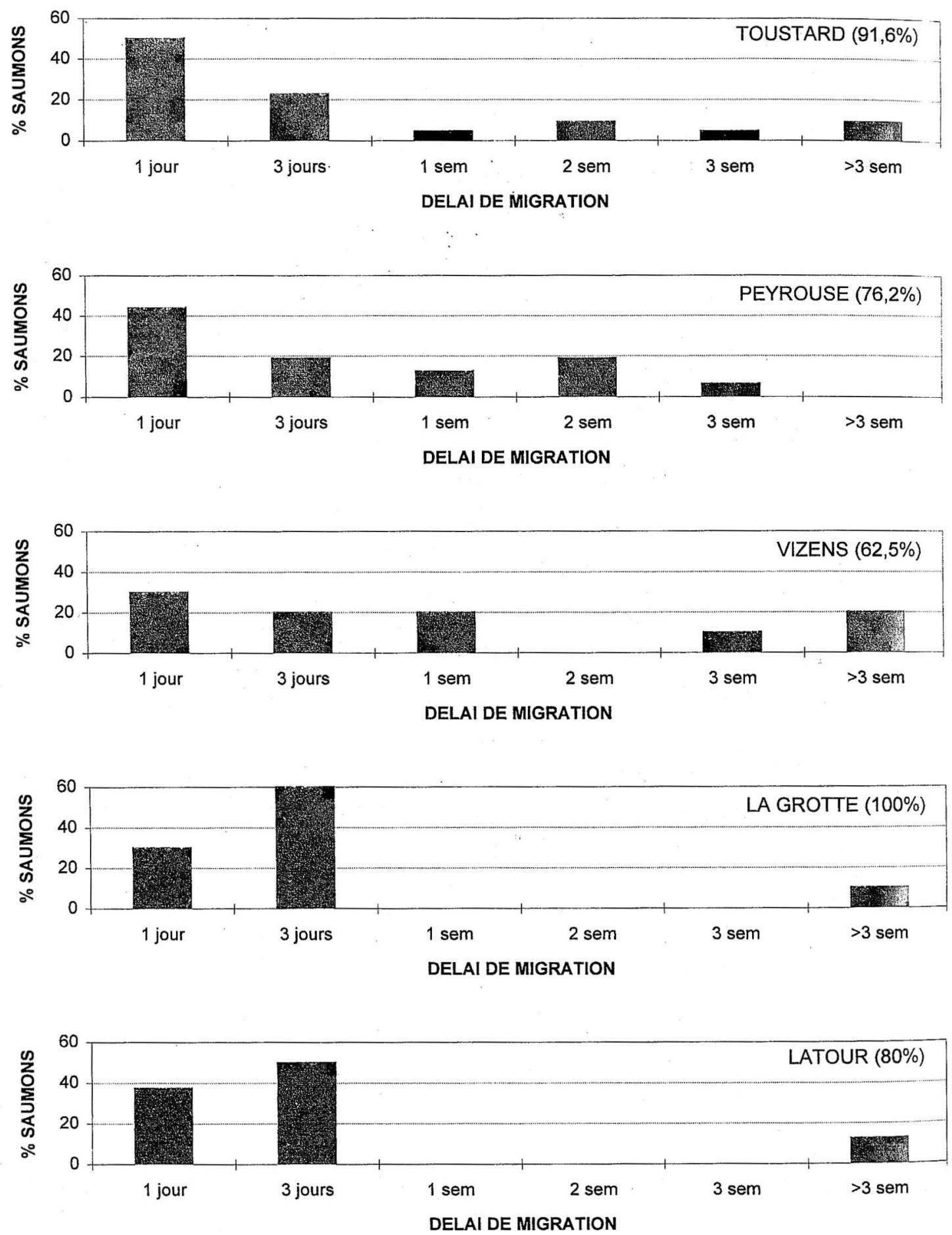

\section{Figure 13}

Délais de migration au niveau des aménagements hydroélectriques situés sur la partie amont du Gave de Pau.

\section{Figure 13}

Migration delays at hydroelectric plant located in the upper part of the river. 

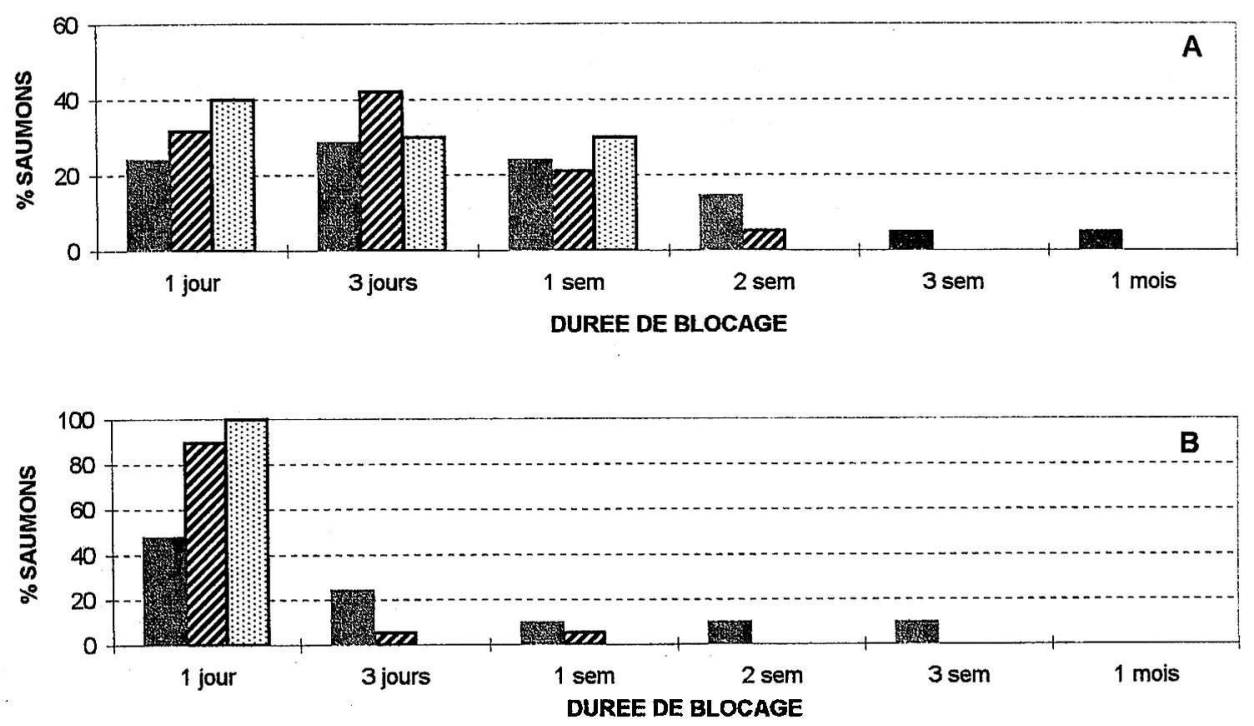

Fiẹure 14A

Délais maximums de migration au niveau de seuils équipés d'une passe à

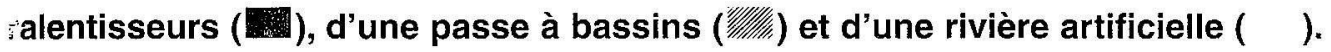

\section{Figure 14B}

Délais minimums de migration au niveau de seuils équipés d'une passe à

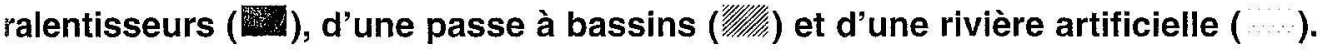

\section{Figure 14A}

Maximum migration delays at weirs fitted with Denil fish pass (橰), pool fish pass (")ilum) and artificial river ( ).

Figure 14B

Minimum migration delays at weirs fitted with Denil fish pass (n), pool fish pass (WIIn) and artificial river ( ).

\section{Au niveau des seuils en enrochements}

II a ainsi été possible de comparer l'efficacité des passes à ralentisseurs, des passes à bassins et des rivières artificielles au niveau des seuils de Denguin, Artiguelouve, Meillon, Narcastet et Assat (Figures 14A et 14B). Ces obstacles, implantés sur une distance de 25 kilomètres, sont de hauteur (entre 2,5 et 3,5 m) et de largeur comparables. lis sont soumis aux mêmes conditions de débit, aucun affluent ne venant augmenter de façon sensible le débit du gave entre les différents obstacles. De plus, les passes sont toutes situées en berge, sur la rive opposée à l'écoulement principal.

L'obstacle équipé d'une rivière artificielle (Meillon) a laissé passer tous les poissons en moins d'une semaine. Quarante pour cent des poissons ont subi des retards inférieurs à une journée. Aucun poisson n'ayant pu être localisé au pied de l'obstacle, tous ont très bien pu franchir l'aménagement en moins de 24 heures.

Les seuils équipés de passe à bassins (Assat et Artiguelouve) ont retardé la majorité des individus moins d'une semaine, près de $90 \%$ d'entre eux ayant pu passer à l'amont en moins d'une journée. Un seul poisson a été retardé plus d'une semaine ; ce retard peut être attribué à une obstruction de la passe, le passage à l'amont de ce poisson étant survenu dans les 24 heures qui ont suivi le nettoyage du dispositif de franchissement. 
Les ouvrages équipés de passes à ralentisseurs (Denguin et Narcastet) ont été pi:s sélectifs $(84 \%)$ et ont entraîné des retards de migration significativement plus importan:s, moins de $50 \%$ des poissons ayant pu franchir ces obstacles en moins de 24 heures. plus, $20 \%$ d'entre eux ont été bloqués au minimum une semaine.

\section{Au niveau d'aménagements hydroélectriques}

Les aménagements de Biron et d'Artix présentent des similitudes tant par leur dé it d'équipement des usines (respectivement $90 \mathrm{~m}^{3} / \mathrm{s}$ et $110 \mathrm{~m}^{3} / \mathrm{s}$ ) que leur configuration $(p: s$ de dérivation). L'obstacle de Biron, équipé d'une rivière de contournement alimentée $r$ un débit de $4 \mathrm{~m} / \mathrm{s}$, a été totalement perméable à la migration, induisant des retards le pi s souvent inférieurs à 24 heures, révélant une très bonne efficacité du dispositif e franchissement. La passe à bassins d'Artix (débit de $750 \mathrm{l} / \mathrm{s}$ plus $2,5 \mathrm{~m}^{3} / \mathrm{s}$ de débit d'attrét) a été moins performante que la rivière de Biron. Elle a permis le passage de la gran ie majorité des poissons mais $35 \%$ des saumons ont été retardés plus de 3 jours. Toutefois, pendant les deux premières années d'étude, le dispositif a été nettement sous-alimen:é (conduite délivrant le débit complémentaire d'attrait en grande partie obturée), ce qui a pu réduire son attractivité et par conséquent augmenter les durées de blocage.

\section{Influence des débits dans les tronçons court-circuités}

L'influence des débits réservés dans les bras court-circuités a été étudiée au niveau de 4 aménagements hydroélectriques (Toustard, Peyrouse, Vizens et Latour) situés sur l'amont du secteur d'étude, comparables tant par leur configuration (longueur et largeur des bras et des canaux de fuite) que par les débits d'équipement des usines, compris entre $24 \mathrm{~m}^{3} / \mathrm{s}$ et $30 \mathrm{~m}^{3} / \mathrm{s}$ (Figure 15). Les horaires de 206 incursions dans les bras court-circuités ou dans les canaux de fuite ont pu être enregistrés ainsi que les débits instantanés turbinés et déversés lors de ces incursions.

Les poissons s'engagent préférentiellement dans les canaux de fuite des aménagements tant que les débits dans les bras court-circuités restent inférieurs à $6 \mathrm{~m}^{3} / \mathrm{s}$, les saumons y pénétrant même près de 7 fois sur 10 lorsque les différents bras ne sont alimentés que par le débit réservé, soit environ $1,2 \mathrm{~m}^{3} / \mathrm{s}$ (Figure 16A). Cette tendance s'inverse pour des débits supérieurs à $6 \mathrm{~m}^{3} / \mathrm{s}$ (soit $14 \%$ du module), valeur qui correspond au niveau de ces aménagements à environ $20 \%$ à $25 \%$ du débit turbiné (Figure 16B).

Si l'on rapporte les débits d'alimentation des bras à leur largeur au niveau de la confluence avec les canaux de fuite, il apparaît que jusqu'à $200 \mathrm{l} / \mathrm{s} / \mathrm{m}$, les poissons ont tendance à remonter préférentiellement dans les canaux, les bras court-circuités devenant nettement plus attractifs lorsque les valeurs dépassent $500 \mathrm{l} / \mathrm{s} / \mathrm{m}$ (Figure 16C).

\section{Conditions de franchissement des passes}

\section{Horaires de franchissement}

Les horaires de 90 franchissements de passes à poissons ont été déterminés. II apparaît que 85,6 \% d'entre eux se sont effectués de jour contre à peine 14,4\% er pleine nuit. En ce qui concerne les franchissements nocturnes, 10 d'entre eux, soit $77 \%$. ont eu lieu au niveau des aménagements équipés d'une rivière de contournement et $\varepsilon$ dans une passe à bassins. 

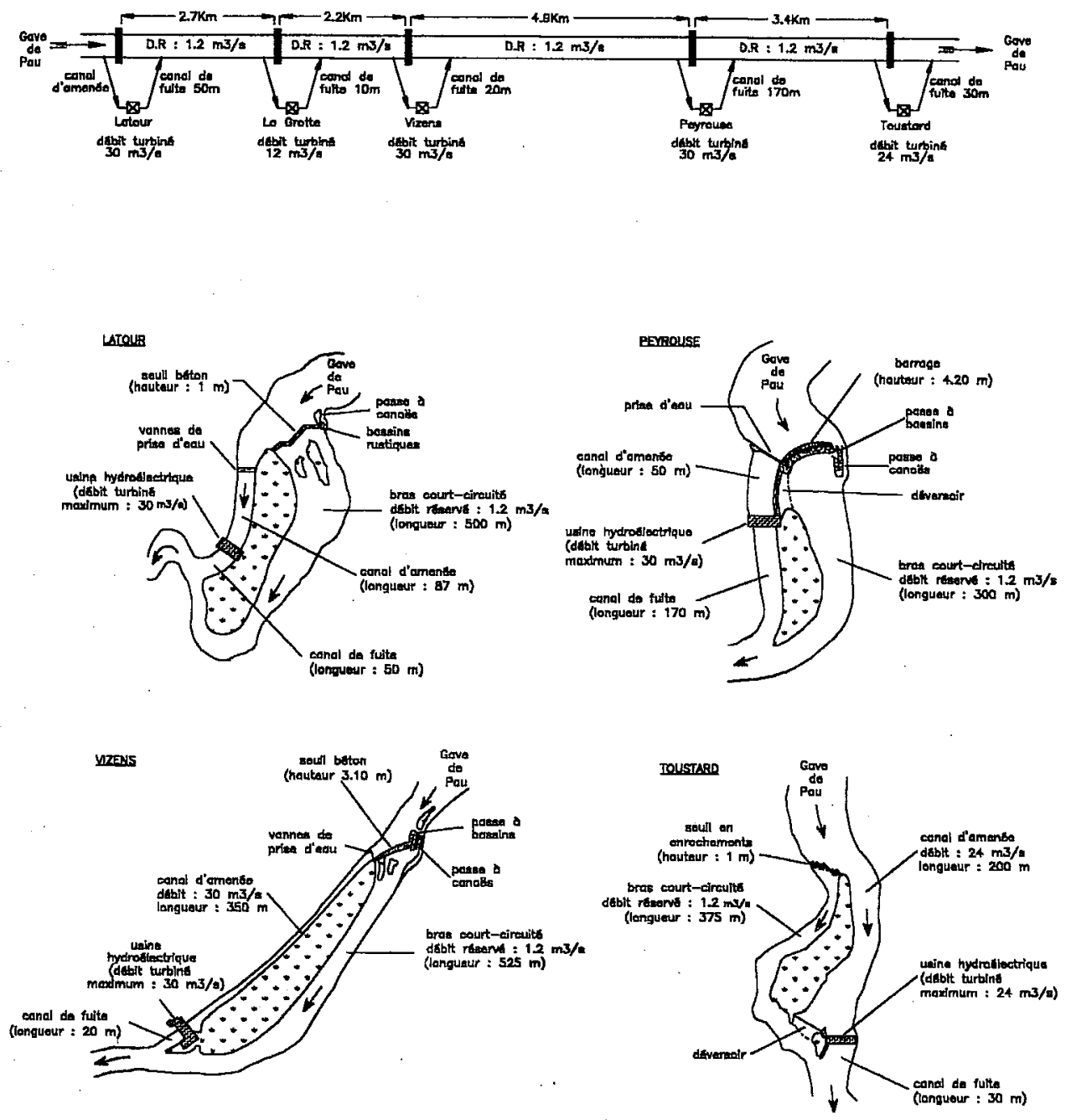

\section{Figure 15}

Présentation des aménagements hydroélectriques munis de dérivation.

\section{Figure 15}

Presentation of hydroelectric plants with derivation.

\section{Durée de franchissement}

Vingt-cinq durées de présence dans les passes à poissons précédant le franchissement d'un obstacle ont été déterminées au cours des 3 années d'étude : 18 d'entre elles concernent les rivières de contournement de Biron et du Lac des Gaves et 7 concernent une passe à bassins.

Les deux rivières de contournement étudiées ne sont comparables, ni par leur situation sur l'axe de migration, ni par leur débit d'alimentation, leur longueur et leur pente. La première (aménagement de Biron) est située sur l'aval du secteur d'étude. Elle est longue de $250 \mathrm{~m}$, est alimentée par un débit de $4 \mathrm{~m}^{3} / \mathrm{s}$ et a une pente moyenne de 1,6\%. Les temps de séjour à l'intérieur du dispositif ont été relativement homogènes : 7 saumons sur 10 ont parcouru les $250 \mathrm{~m}$ en moins de $1 \mathrm{~h} \mathrm{40,} \mathrm{ce} \mathrm{qui} \mathrm{correspond} \mathrm{à} \mathrm{des} \mathrm{vitesses} \mathrm{de}$ déplacement comprises entre $0,14 \mathrm{~km} / \mathrm{h}$ et $0,25 \mathrm{~km} / \mathrm{h}$. Trois poissons enfin ont été présents environ 2 h 30 dans la rivière, ce qui correspond à des vitesses moyennes de lordre de $0,10 \mathrm{~km} / \mathrm{h}$. 

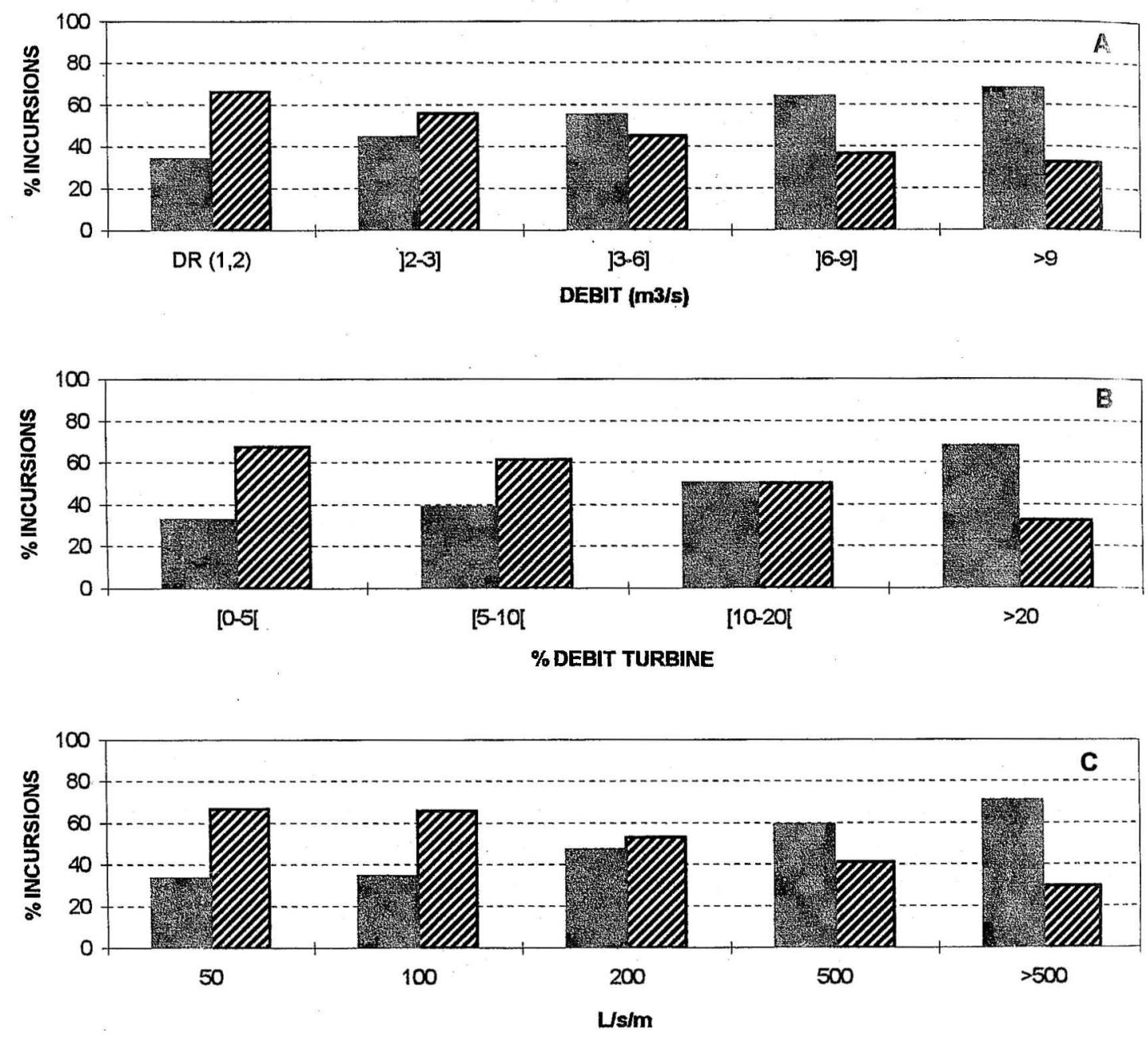

Figure 16A

Remontées des poissons dans les bras court-circuités (19 canaux de fuitı (Vivin) en fonction du débit $\left(\mathrm{m}^{3} / \mathrm{s}\right)$ dans les bras court-circuités.

Figure 16B

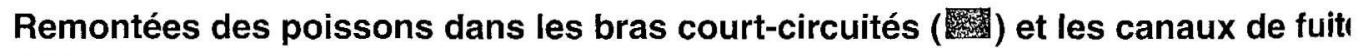
(Viv/u) en fonction du pourcentage du débit dans les bras court-circuités pa rapport au débit turbiné.

Figure 16C

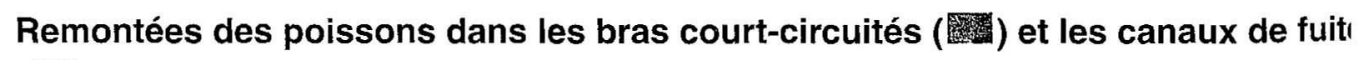
(Velle) en fonction du débit $\left(\mathrm{m}^{3} / \mathrm{s}\right)$ par mètre de largeur des bras court-circuités.

\section{Figure 16A}

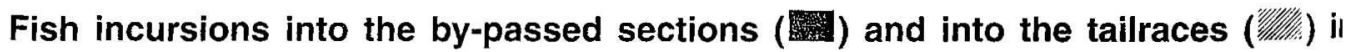
relation with discharge $\left(\mathrm{m}^{3} / \mathrm{s}\right)$ in the by-passed sections.

\section{Figure 16B}

Fish incursions into the by-passed sections (嫼) and into the tailraces ( relation with discharge percentage in the by-passed sections compared $t$ discharge in the tailraces.

\section{Figure 16C}

Fish incursions into the by-passed sections (17.7.7. relation with discharge $\left(\mathrm{m}^{3} / \mathrm{s}\right)$ per meter of width in the by-passed sections. 
La rivière artificielle du Lac des Gaves est située sur la partie amont du secteur d'stude, 100 kilomètres à l'amont de l'aménagement de Biron. Elle est longue de $1,2 \mathrm{~km}$, es alimentée par un débit de $1,5 \mathrm{~m}^{3} / \mathrm{s}$ et a une pente moyenne de $1 \%$. Les 8 ranchissements n'ont concerné que deux poissons, un saumon ayant remonté puis $\mathrm{d}$ scendu la rivière plusieurs fois. A cinq reprises, les poissons ont mis entre $2 \mathrm{~h} 30$ et $4: 00$ pour parcourir les $1,2 \mathrm{~km}$ de la rivière, ce qui correspond à des vitesses moyennes di déplacement comprises entre $0,29 \mathrm{~km} / \mathrm{h}$ et $0,47 \mathrm{~km} / \mathrm{h}$. Les durées de franchissement le. plus importantes ( $8 \mathrm{~h} 40$ et 10 h 10) ont été observées lorsque les poissons ont pénétré à intérieur du dispositif de franchissement en pleine journée.

La passe à fentes verticales de l'aménagement d'Artix est composée de 22 bassins et əst alimentée par un débit de $750 \mathrm{l} / \mathrm{s}$. Les durées de présence de 7 saumons dans la p s se ont pu être déterminées. Cinq poissons ont mis moins de 2 heures pour parcourir le: 19 premiers bassins, ce qui correspond à des durées moyennes de stationnement par $b_{c}$ sin comprises entre $3 \mathrm{mn}$ et $6 \mathrm{mn}$. Un poisson a séjourné en moyenne près de $21 \mathrm{mn}$ pe: bassin pour un temps total de présence dans la passe supérieur à 6,5 heures. $\mathrm{Ce}$ saumon a été le seul à s'engager de nuit à l'intérieur du dispositif de franchissement.

\section{Températures lors des franchissements}

La très grande majorité des entrées dans les passes a été observée pour des températures de l'eau comprises entre $9^{\circ} \mathrm{C}$ et $20^{\circ} \mathrm{C}$. Onze franchissements se sont efíectués pour des températures de l'eau supérieures à $20^{\circ} \mathrm{C}$ avec un maximum de $24,4^{\circ}$ C. Seules 4 entrées à l'intérieur des dispositifs se sont effectuées pour des températures inférieures à $9^{\circ} \mathrm{C}$ (minimum $7,5^{\circ} \mathrm{C}$ ), 3 d'entre elles se produisant dans une rivière artificielle.

\section{Dévalaison des dispositifs}

Des dévalaisons d'obstacles par le dispositif de franchissement ont pu être mis en évidence uniquement au niveau de la rivière de contournement du Lac des Gaves. Les horaires de 8 descentes de la rivière dont 7 suivies d'une remontée du dispositif de franchissement ont été enregistrés. La grande majorité de ces dévalaisons a été effectuée de nuit ou au crépuscule, une seule d'entre elles, la plus longue (près de $12 \mathrm{~h} \mathrm{00}$ ), ayant débuté en pleine journée. A 5 reprises, les temps ont été inférieurs à $2 \mathrm{~h} 15$, ce qui correspond à des vitesses de dévalaison comprises entre $0,54 \mathrm{~km} / \mathrm{h}$ et $2 \mathrm{~km} / \mathrm{h}$.

\section{DISCUSSION}

\section{Rythmes de migration}

Les déplacements des poissons sur le Gave de Pau sont, à l'heure actuelle, entièrement artificialisés. Les arrêts de migration s'effectuent généralement au pied des obstacles, les poissons nageant jusqu'à l'aménagement suivant après avoir franchi un ouvrage. Ce sont plus les obstacles et leur difficulté de franchissement que les paramètres de débit et de température qui rythment la remontée des saumons. Toutefois, lors des 3 années d'étude, les températures de l'eau ont été la plupart du temps comprises entre $7^{\circ} \mathrm{C}$ et $20^{\circ} \mathrm{C}$, plage de valeurs permettant aux saumons d'exprimer pleinement leur comportement migratoire (ALABASTER, 1990 ; JONSSON, 1991 ; CRISP, 1996). Entre les aménagements, les vitesses de déplacement, comprises entre $0,3 \mathrm{~km} / \mathrm{h}$ et $1,6 \mathrm{~km} / \mathrm{h}$, sont du même ordre de grandeur que les valeurs couramment observées sur des portions non obstruées de cours d'eau (HAWKINS et SMITH, 1986 ; LAUGHTON, 1989). 
Un des rares phénomènes naturels à avoir pu être observé est l'arrêt esti al de migration (WEBB, 1989 ; LAUGHTON, 1991 ; WEBB, 1992). Les arrêts mis in évidence, compris entre 40 jours et 75 jours, sont généralement précédés d'une ne augmentation des températures de l'eau associée à une baisse des débits. Trois d'ent: $e$ eux sont survenus après que les poissons se soient présentés au pied d'un obstacle. $L: S$ reprises de migration, au début de l'automne, se produisent suite à de fortes diminutio s des températures.

\section{Franchissabilité des ouvrages et restauration des stocks}

Les trois années d'étude ont permis de mettre en évidence l'impact de 31 sur $a$ migration anadrome des poissons des 38 obstacles construits sur le cours d'eau, ; d'obtenir une vision globale de l'ensemble de l'axe migratoire pour une distance totale plus de 126 kilomètres de cours d'eau. Un classement des obstacles a pu être effectué $\epsilon$ ? intégrant la connaissance et l'expertise des différents sites, leur position sur l'axe, le; retards engendrés, les pourcentages de franchissement et le nombre de poissons stir lequel reposent ces observations.

Seize aménagements n'ont pas présenté de réelles difficultés de franchissemeni, laissant passer la quasi-totalité des poissons dans des délais très souvent inférieurs à 3 jours. Dix ouvrages constituent de plus sérieux obstacles à la migration en termes de blocage sur une partie de la population migrante et/ou de retards notables dans la migration. Cinq aménagements enfin représentent des obstacles majeurs à la migration des poissons, ces ouvrages étant sélectifs et induisant des retards de migration importants pouvant dépasser une cinquantaine de jours. L'un d'entre eux (Baigts) est incompatible avec tout plan de restauration en regard de sa forte sélectivité (35\% des poissons sont passés à l'amont), des délais de migration qu'il induit (jusqu'à 79 jours) et de sa position très aval sur l'axe.

En l'état actuel des choses, si l'on ne tient compte que de la sélectivité des différents obstacles érigés sur l'axe, $13 \%$ des saumons peuvent parvenir à l'amont de la ville de $\mathrm{Pau}$, sur de bonnes zones de frayères, et $3 \%$ à peine peuvent atteindre les meilleures zones situées à l'amont de la ville de Nay.

On peut raisonnablement penser que les problèmes de franchissement seront résolus sur les obstacles majeurs, comme ils l'ont été récemment au barrage d'Artix, les causes des dysfonctionnements ayant été identifiées. Dans l'hypothèse où ces aménagements seraient transparents, $80 \%$ des poissons parviendraient à l'amont de Pau et $50 \%$ à l'amont de Nay.

$\mathrm{Si}$, de plus, on considère les retards induits par tous les aménagements, un certain nombre de poissons ne pourra arriver à temps sur les frayères, notamment ceux qui se présentent tardivement sur le gave, pendant les mois de septembre et d'octobre (près de $30 \%$ des poissons lors des 3 années), certains saumons ayant en effet mis plus de 4 mois pour atteindre la ville de Pau. Le même problème se pose pour les poissons qui, retardés par les aménagements situés sur l'aval du secteur d'étude, effectuent un arrêt estival jusqu'à l'automne sur les parties basse et moyenne du secteur d'étude. Il convient donc également de réduire les retards à la migration, en particulier au niveau des obstacles situés à l'aval des meilleures zones de frayères, en modifiant ou complétant les aménagements actuels.

\section{Efficacité des dispositifs de franchissement}

Les différents dispositifs de franchissement étudiés ont présenté des performances très variables selon le type d'ouvrage sur lesquels ils ont été construits. 
Au niveau des usines hydroélectriques ne comportant pas de dérivation, l'efficacité d $\epsilon$ s dispositifs de franchissement dépend plus de leur implantation et de leur débit que du ty $e$ de passe. Des dispositifs dont l'entrée, située dans le canal de fuite, mobilise des dojits importants $\left(2-4 \mathrm{~m}^{3} / \mathrm{s}\right)$, permettent le passage de la très grande majorité des pi ssons et n'engendrent que de faibles retards de migration. La majorité des fru achissements dans les passes à bassins s'effectue en pleine journée, comme il l'a été c istaté sur d'autres cours d'eau (DARTIGUELONGUE et LANGON, 1998 ; GOWANS, A MSTRONG et PRIEDE, 1999). Par contre, une fraction importante des franchissements $d s$ rivières de contournement s'effectue de nuit, ces dispositifs paraissant ainsi respecter di rantage les rythmes naturels des déplacements des saumons, principalement nocturnes (k) LNER, 1990 ; SMITH et SMITH, 1997).

Les perméabilités des différents aménagements comportant une dérivation di jendent autant des conditions de débit dans les parties court-circuitées que des d positifs de franchissement (type et implantation) équipant ces ouvrages.

Au niveau des seuils en enrochements, les dispositifs de franchissement ont pissenté des efficacités variables. Les passes à ralentisseurs à chevrons épais, installées a ciant pour le canoë-kayak que pour le poisson sur les premiers seuils aménagés sur le give, ne paraissent adaptées ni à ce type d'obstacle, ni à un cours d'eau comme le Gave do: Pau, comme l'avait déjà noté LARINIER (1992). Ces dispositifs, installés le plus scitvent en berge au niveau des seuils dont la largeur est importante (plus de $90 \mathrm{~m}$ ), trensitent des débits faibles rendant leur attractivité insuffisante. Elles sont très sensibles atı $x$ variations du niveau d'eau amont et souvent sujettes à des obstructions.

Les passes à bassins à gros débit ( $>1 \mathrm{~m}^{3} / \mathrm{s}$ en étiage) et les rivières de contournement ont été nettement plus performantes que les passes à ralentisseurs, laissant passer la quasi-totalité des poissons dans des délais acceptables, la plupart inférieurs à 3 jours. Elles sont de plus beaucoup moins sensibles aux variations des niveaux d'eau.

II n'en reste pas moins vrai que, de tous les obstacles étudiés sur le gave, ceux qui ont eu l'impact le plus faible sur la migration des saumons ont été les seuils en enrochements de hauteur inférieure à 1,2-1,5 $\mathrm{m}$, non équipés de dispositif de franchissement. Aucun poisson n'a d'ailleurs pu être localisé au pied de ces ouvrages lors des 3 années d'étude. Ce type d'aménagement limite par ailleurs considérablement les problèmes d'entretien qui s'avèrent être l'un des problèmes majeurs des dispositifs de franchissement implantés sur les seuils en enrochements (LARINIER, 1992).

\section{Influence des débits dans les tronçons court-circuités}

Des débits de l'ordre de $6 \mathrm{~m}^{3} / \mathrm{s}$ (soit $14 \%$ du module au niveau des obstacles étudiés) dans les tronçons court-circuités, valeur qui correspond à 20-25\% du débit turbiné maximum, sont apparus nécessaires pour augmenter de façon significative l'attractivité de ces bras court-circuités, les poissons remontant préférentiellement dans ces conditions au pied des barrages alors que les usines fonctionnent pourtant à plein régime.

Les valeurs obtenues ne sont a priori valables que pour les aménagements étudiés, caractérisés par des débits d'équipement de l'ordre de $30 \mathrm{~m}^{3} / \mathrm{s}$ et des largeurs des canaux de fuite et des bras voisins respectivement de $10-15 \mathrm{~m}$ et $25 \mathrm{~m}$. Si l'on rapporte les débits d'alimentation des bras à leur largeur au niveau de la confluence avec les canaux de fuite, il apparaît que jusqu'à $200 \mathrm{l} / \mathrm{s} / \mathrm{m}$, les poissons ont tendance à remonter préférentiellement dans les canaux, les bras court-circuités devenant nettement plus attractifs lorsque les valeurs dépassent $500 \mathrm{l} / \mathrm{s} / \mathrm{m}$. 


\section{CONCLUSION}

On peut être raisonnablement optimiste sur les possibilités de réouverture du Ge /e de Pau à la migration du saumon en améliorant la transparence des obstacles majel:s identifiés lors de l'étude. Le système n'en demeure pas moins fragile et il conviendra de le maintenir sous haute surveillance, en particulier en ce qui concerne l'entretien is dispositifs de franchissement. Présence de dérivations, débits réservés insuffisar $s$, piégeage dans les canaux de fuite des usines, défaut d'entretien ou de maintenance $c$ is dispositifs, sont autant de sources supplémentaires de retards ou de blocage.

Cette étude illustre par ailleurs plusieurs principes souvent énoncés dans :s ouvrages ou manuels techniques traitant des dispositifs de franchissement, principes u tiennent du bon sens et de l'expertise mais qui sont rarement étayés par des exempis concrets. Elle montre en particulier clairement que la notion d'efficacité d'un dispositif : $\epsilon$ franchissement recouvre non seulement le pourcentage de franchissement mais aussi ie retard dans la migration induit par l'obstacle. Un cours d'eau comportant de nombre:ix obstacles, même équipé de dispositifs de franchissement performants, peut induire des retards dont le cumul est susceptible de mettre en péril la réussite d'un plan de restauration.

\section{REMERCIEMENTS} E. GALIAY.

Les études sur le terrain ont bénéficié de l'aide technique de J.M. BACH e

Nous remercions tous les producteurs autonomes qui nous ont permis d'installer $d \mathrm{c}$ matériel dans leurs locaux ainsi que la SHEM et EDF, en particulier les groupements de Lau Balagnas et de Baigts, les Fédérations des Hautes-Pyrénées et des Pyrénées-Atlantiques pour la Pêche et la Protection du Milieu Aquatique, l'AAPPMA de Lourdes et MIGRADOUR.

\section{BIBLIOGRAPHIE}

ALABASTER J.S., 1990. The temperature requirements of adult Atlantic salmon, Salme salar L., during their upstream migration in the River Dee. J. Fish Biol., 37, 659-661

CRISP D.T., 1996. Environmental requirements of common riverine European salmonic fish species in fresh water with particular reference to physical and chemica aspects. Hydrobiologia, 323, 201-221.

DARTIGUELONGUE J., LANGON M., 1998. Contrôle du fonctionnement de l'ascenseur ì poissons de Tuilières en 1997. Suivi de l'activité ichtyologique. Rapport S.C.E.A pour MI.GA.DO., $33 \mathrm{p}$.

GOWANS A.R.D., ARMSTRONG J.D., PRIEDE 1.G., 1999. Movements of adult Atlantic salmon in relation to a hydroelectric dam and fish ladder. J. Fish. Biol., 54, 713-726

HAWKINS A.D., SMITH G.W., 1986. Radio-tracking observations on Atlantic salmor ascending the Aberdeenshire Dee. Scottish Fisheries Research Report, 36, 24 p.

JONSSON N., 1991. Influence of water flow, water temperature and light on fish migratior in rivers. Nordic J. Freshw. Res., 66, 20-35.

LARINIER M., 1992. Le franchissement des buses, des seuils en enrochements et de! ouvrages estuariens. Bull. Fr. Pêche Pisc., 326-327, 111-124.

LAUGHTON R., 1989. The movements of adult salmon within the River Spey. Scottis/ Fisheries Research Report, 41, $19 \mathrm{p}$. 
LAUGHTON R., 1991. The movements of adult Atlantic salmon (Salmo salar L.) in the River Spey as determined by radiotelemetry during 1988 and 1989. Scottish Fisheries Research Report, 50, 34 p.

MRTY A., 1984. Le saumon dans les bassins de l'Adour et de la Nivelle. Rapport d'étude. Conseil Supérieur de la Pêche, Délégation régionale Midi-Pyrénées, 187 p.

M NER N.J.(ed.), 1990. Fish movements in relation to fresh water flow and quality. Proceedings of Atlantic Salmon Trust/Wessex Water Workshop, Pitlochry, Atlantic Salmon Trust, $51 \mathrm{p}$.

S! TH I.P., SMITH G.W., 1997. Tidal and diel timing of river entry by adult Atlantic salmon returning to the Aberdeenshire Dee, Scotland. J. Fish Biol., 50, 463-474.

Si. TH G.W., CAMPBELL R.N.B., MACLAINE J.S., 1998. Regurgitation rates of intragastric transmitters by adult Atlantic salmon (Salmo salar L.) during riverine migration. Hydrobiologia, 371/372, 117-121.

SC.OMON D.J., STRORETON-WEST T.J., 1983. Radio-tracking of migratory salmonids in rivers : development of an effective system. Ministry of Agriculture, Fisheries and Food, Directorate of Fisheries Research. Fisheries Research technical Report, 75, $11 \mathrm{p}$.

STASKO A.B., PINCOCK D.G., 1977. Review of underwater biotelemetry, with emphasis on ultrasonic techniques. J. Fish. Res. Board Can., 34, 1261-1285.

WEBB J., 1989. The movements of adult Atlantic salmon in the River Tay. Scottish Fisheries Research Report, 44, $32 \mathrm{p}$.

WEBB J., 1992. The behaviour of adult salmon (Salmo salar L.) in the River Tay as determined by radiotelemetry. Scottish Fisheries Research Report, 52, $19 \mathrm{p}$. 